Max-Planck-Institut für demografische Forschung

Max Planck Institute for Demographic Research

Konrad-Zuse-Strasse 1 - D-18057 Rostock · GERMANY

Tel +49 (0) 3812081 - 0; Fax +49 (0) 3812081 - 202;

http://www.demogr.mpg.de

MPIDR WORKING PAPER WP 2006-022

AUGUST 2006

Fertilität, Familiengründung und

Familienerweiterung in den nordischen Ländern

Gerda Neyer (neyer@demogr.mpg.de)

Gunnar Andersson (andersson@ demogr.mpg.de)

Jan Hoem (hoem@ demogr.mpg.de)

Marit Rønsen

Andres Vikat

(C) Copyright is held by the authors.

Working papers of the Max Planck Institute for Demographic Research receive only limited review. Views or opinions expressed in working papers are attributable to the authors and do not necessarily reflect those of the Institute. 


\title{
Fertilität, Familiengründung und Familienerweiterung in den nordischen Ländern
}

\author{
Gerda Neyer ${ }^{1}$, Gunnar Andersson ${ }^{1}$, Jan Hoem ${ }^{1}$, Marit Rønsen ${ }^{2}$, Andres Vikat $^{1}$
}

Zusammenfassung: Dieser Beitrag beschäftigt sich mit der Geburtenentwicklung in den nordischen Ländern seit den 1970er Jahren und den Wirkungen familienpolitischer Maßnahmen auf die Fertilitätsentwicklung. Basis der Analysen bilden Auswertungen harmonisierter Registerdaten Dänemarks, Finnlands, Norwegens und Schwedens. Der erste Teil des Beitrags bietet einen Überblick über die Entwicklung der Fertilität in den nordischen Ländern nach Alter und Geburtenordnung. Dies erlaubt, gemeinsame von länderspezifischen Entwicklungen zu unterscheiden. Daran schließt sich eine Darstellung des Zusammenhangs zwischen Bildungsrichtungen und Fertilität. Im letzten Teil des Beitrages erörtern wir, welchen Einfluss familienpolitische Maßnahmen, insbesondere ein einkommensbezogenes Elterngeld, ein auf den Geburtenabstand bezogenes Elterngeld, sowie die Inanspruchnahme der Elternzeit durch Väter auf Geburtenverhalten und Geburtenentwicklung in den einzelnen Ländern hatten.

\footnotetext{
${ }^{1}$ Max-Planck-Institut für demografische Forschung, Rostock

${ }^{2}$ Statistics Norway, Oslo
} 


\section{Einleitung ${ }^{1}$}

Wie in allen westeuropäischen Ländern ist auch in den nordischen Staaten ${ }^{2}$ die Geburtenrate seit den 1960er Jahren deutlich gesunken. 1965 lag die Gesamtfertilitätsrate ${ }^{3}$ in den nordischen Ländern noch zwischen 2.95 (Norwegen) und 2.42 (Schweden), in Deutschland bei 2.50. ${ }^{4}$ Im Jahre 2004 reichte sie von 1.75 (Schweden) bis 1.83 (Norwegen) (Deutschland $1.36^{5}$ ) und lag damit, wie in allen europäischen Ländern, unter dem Reproduktionsniveau von 2.1. Doch im Gegensatz zu Deutschland, dessen Gesamtfertilitätsrate auch nach dem starken Rückgang in den späten 1960er und frühen 1970er Jahren tendentiell leicht rückläufig blieb, verzeichneten die nordischen Länder in den 1980er und 1990er Jahren zum Teil steigende Gesamtfertilitätsraten (Grafik A1 im Anhang). ${ }^{6}$ Diese scheinen sich auf einem im europäischen Vergleich relativ hohen Niveau zu stabilisieren. Norwegen, Finnland, Dänemark und - mit Ausnahme der zweiten Hälfte der 1990er Jahre - auch Schweden zählen zu den Ländern mit der höchsten Fertilität in Europa - Deutschland dagegen zu den Ländern mit der niedrigsten (Council of Europe 2004).

Die Unterschiede in der Entwicklung der Fertilität zwischen den nordischen Ländern und Deutschland sind nicht allein auf periodenspezifische Differenzen im Geburtenverhalten der Frauen dieser Länder zurückzuführen. Frauen in Deutschland gebären im Laufe ihres Lebens weniger Kinder als Frauen in den nordischen Ländern. Die zwischen 1945 und 1960 geborenen Frauen Deutschlands weisen sogar die niedrigsten vollständigen Kohortenfertilitätsraten ${ }^{7}$ Europas auf (Council of Europe 2004). Die Differenz zwischen der Kohortenfertilität der nordischen Länder und jener Deutschlands hat sich über die nach 1945

1 Die vorliegende Arbeit ist im Rahmen des „Nordic Network“ entstanden, einer Forschungskooperation, der neben den AutorInnen auch Kari Skrede, Trude Lappegård (Statistics Norway) und Lisbeth Knudsen (Aalborg University) angehören. Die Zusammenarbeit wurde durch das Welfare Research Programme of the Nordic Council of Ministers (2002-2005) unterstützt. Wir danken Kari Skrede, Trude Lappegård, Lisbeth Knudsen, Merethe Johansen (Familienministerium Dänemark) und Anne Hornung für Informationen, Kommentar und Unterstützung für diesen Beitrag.

${ }^{2}$ Unter den nordischen Staaten fassen wir die skandinavischen Staaten (Dänemark, Norwegen, Schweden) und Finnland zusammen. Auf Island wird in diesem Bericht nicht direkt eingegangen, da wir über keine Registerdaten dieses Landes verfügten und somit Island nicht in die vergleichende Analyse der Fertilitätsentwicklung in den nordischen Ländern (siehe Abschnitt 2) einbeziehen konnten.

${ }^{3}$ Die Gesamtfertilitätsrate wird häufig auch als Periodenfertilitätsrate oder als zusammengefasste Geburtenziffer bezeichnet.

4 Soferne nicht ausdrücklich darauf hingewiesen wird, bezeichnet Deutschland immer Ost- und Westdeutschland. 1965 lag die zusammengefasste Geburtenziffer in Ostdeutschland bei 2.48, jene Westdeutschlands bei 2.51 .

${ }^{5}$ In Westdeutschland lag die zusammengefasste Geburtenziffer 2002 bei 1.35, in Ostdeutschland bei 1.06.

${ }^{6}$ Auch in Ostdeutschland stieg die Gesamtfertilitätsrate in der zweiten Hälfte der 1970er Jahre deutlich an (1975: 1.54, 1980: 1.94) und blieb bis zur Wiedervereinigung im Jahre 1990 höher als in Westdeutschland. Der Anstieg und das höhere Gesamtfertilitätsniveau in Ostdeutschland ab Mitte der 1970er Jahre werden von der Forschung der Familien- und Sozialpolitik der DDR zugeschrieben (Trappe 1995, Kreyenfeld 2004b). 
geborenen Frauengenerationen hinweg vergrößert. Die 1945 geborenen Frauen in Deutschland brachten im Schnitt 1.80 Kinder zur Welt; jene in den nordischen Ländern zwischen 1.88 (Finnland) und 2.21 (Norwegen). Für die 1960 geborenen Frauen Deutschlands liegt die vollständige Kohortenfertilitätsrate im Jahre 2004 bei 1.65; in den nordischen Ländern zwischen 1.90 (Dänemark) und 2.09 (Norwegen) (Grafik A2 im Anhang).

Neben ihrer hohen Fertilität zeichnen sich die nordischen Länder bereits seit den 1960er und frühen 1970er Jahren durch einen hohen Anteil an erwerbstätigen Frauen aus. 2004 waren in den nordischen Ländern zwischen 72\% (Finnland) und 76.6\% (Schweden) aller Frauen erwerbstätig ${ }^{8}$, in Deutschland 66.1\%. Der Anstieg der Frauenerwerbstätigkeit hatte in den nordischen Ländern deutlich früher eingesetzt als in Westdeutschland. Zu Beginn der 1960er Jahre entsprach der Anteil der erwerbstätigen Frauen in Westdeutschland (49.2\%) noch dem in Schweden (50.0\%) und lag deutlich über jenem in Dänemark (43.7\%). Doch bereits Mitte der 1960er Jahre bzw. 1970er Jahre waren in den nordischen Ländern weit mehr als die Hälfte aller Frauen im erwerbsfähigen Alter erwerbstätig. In Westdeutschland hingegen verharrte die Frauenerwerbsquote bis Anfang der 1980er Jahre unter 50\%. Erst zu Beginn der 1990er Jahre - rund 20 Jahre später als in Schweden und Dänemark und 12 Jahre später als in Norwegen - stieg der Anteil der erwerbstätigen Frauen in Deutschland auf über 60\%. Dieser Anstieg war in erster Linie auf die Wiedervereinigung Ost- und Westdeutschlands und die damals rund 35 Prozentpunkte höhere Erwerbsquote ostdeutscher Frauen zurückzuführen. Erst im Verlaufe der 1990er Jahre erreichte die Frauenerwerbsquote auch in Westdeutschland $60 \%$ und damit ein Niveau, das eine gesamtgesellschaftliche Änderung des Erwerbsverhaltens von Frauen und potentiell auch des Geschlechterarrangements in der Verteilung von Arbeit signalisiert (Grafik A3 im Anhang).

In den nordischen Ländern war somit der Rückgang der Fertilität in den 1960er und frühen 1970er Jahren zum Teil von einem Anstieg der Frauenerwerbstätigkeit begleitet. Dies entsprach dem für viele westliche Industriestaaten der 1960er Jahre kennzeichnenden Zusammenhang zwischen steigender Frauenerwerbstätigkeit und sinkender Kinderzahl (Brewster/Rindfuss 2000). Ein solcher Zusammenhang wird häufig auch für die nachfolgenden Jahrzehnte angenommen. Doch trotz zunehmender Frauenerwerbstätigkeit ab den 1970er Jahren sank die Fertilität in den nordischen Ländern langfristig nicht weiter ab, sondern stieg und pendelte sich auf einem relativ hohen Niveau ein. In Westdeutschland hingegen erfolgte der Rückgang der Fertilität in den 1960er Jahren ohne signifikanten Anstieg der Frauenerwerbstätigkeit; die Zunahme der Frauenerwerbstätigkeit ab den 1980er Jahren war jedoch von weiterhin fallenden Fertilitätsraten begleitet.

Diese unterschiedlichen Entwicklungsverläufe legen einen möglichen Zusammenhang zwischen der Ausgestaltung der Wohlfahrtsstaaten, der Familienpolitik und der Fertilität nahe.

\footnotetext{
${ }^{7}$ Die vollständigen Kohortenfertilitätsraten geben die durchschnittliche Anzahl der Kinder an, die Frauen eines spezifischen Geburtsjahrganges im Laufe ihres reproduktiven Lebens zur Welt gebracht haben.
} 
Internationale Studien vermuten ebenfalls, dass die institutionellen Rahmenbedingungen in den einzelnen Ländern einen entscheidenden Faktor für die Fertilitätsentwicklung und das Fertilitätsniveau darstellen (Ahn/Mira 2002; Engelhardt/Kögel/Prskawetz 2004; Adserà 2004). Doch Analysen von Aggregatdaten liefern nur Hinweise über mögliche Einflüsse von institutionellen und familienpolitischen Maßnahmen auf Fertilitätsverhalten. Genauere Rückschlüsse über die Wirkungen von Familienpolitik auf Fertilitätsverhalten liefern Analysen von Individualdaten.

Dieser Beitrag zielt daher darauf ab, die Entwicklung der Fertilität in den nordischen Ländern und deren Zusammenhang zu den wohlfahrtsstaatlichen und familienpolitischen Ausprägungen auf der Basis harmonisierter Individualdaten darzustellen. Im ersten Teil des Beitrages bieten wir einen Überblick über die Entwicklung der Fertilität in den nordischen Ländern nach Alter und Parität (Kinderzahl). Dies erlaubt eine differenziertere Einschätzung der Geburtenentwicklung in den letzten Jahrzehnten. Daran schließt sich eine Darstellung des Zusammenhangs zwischen Bildung und Fertilität. Im letzten Teil des Beitrages erörtern wir, welchen Einfluss wohlfahrtsstaatliche und familienpolitische Faktoren auf die Fertilitätsentwicklung der einzelnen Länder haben.

\section{Fertilitätsentwicklung in den nordischen Ländern}

Die Gesamtfertilitätsraten der nordischen Länder weisen, wie in der Einleitung dargestellt, einige Gemeinsamkeiten auf. Nach einem deutlichen Rückgang in den 1960er und 1970er Jahren stiegen die Fertilitätsraten in den 1980er und frühen 1990er Jahren in allen Ländern an. Besonders ausgeprägt war dieser Anstieg in Schweden. Dort stieg die Periodenfertilitätsrate von 1.61 im Jahre 1983 auf 2.13 im Jahre 1990, fiel danach jedoch auf ein historisches Tief von 1.50 in den Jahren 1998 und 1999. Seither steigt die Gesamtfertilitätsrate wieder an (2002: 1.65; 2003: 1.71) und näherte sich 2005 mit 1.77 wieder dem nordischen Durchschnitt. Weit stabiler verlief die Entwicklung in den anderen nordischen Ländern. In Finnland schwankte die Fertilitätsrate zwar ebenfalls, jedoch in deutlich geringerem Ausmaß als in Schweden. Dänemark und Norwegen verzeichneten demgegenüber eine gleichmäßigere Entwicklung. In beiden Ländern stieg die Fertilitätsrate in den 1980er Jahre deutlich an. In Dänemark, das 1983 nach Westdeutschland die niedrigste Fertilität in Europa aufwies, war dieser Anstieg ausgeprägter und länger dauernd als in Norwegen, dessen Gesamtfertilitätsrate sich allerdings bereits Anfang der 1980er Jahre auf einem vergleichsweise hohen Niveau bewegte. In Summe waren in der letzten Dekade die Gesamtfertilitätsrate Norwegens, Finnlands und Dänemark relativ stabil und lag zwischen 1.89 (Norwegen) und 1.70 (Finnland). In Schweden dagegen fluktuierte sie deutlich.

Die Gesamtfertilitätsrate spiegelt die Entwicklung der Fertilität jedoch nur unzureichend wider. Zwei Aspekte sind für unsere Betrachtungen besonders von Bedeutung: Zum einen können Tempoeffekte die Gesamtfertilitätsrate beeinflussen. Bei einem Aufschub

\footnotetext{
${ }^{8}$ Die Angaben beziehen sich auf Frauen im erwerbsfähigen Alter (15 bis 64).
} 
der Geburten, häufig ablesbar an einem steigenden Durchschnittsalter von Frauen bei einer (Erst)Geburt, sinkt die Gesamtfertilitätsrate, ohne dass dies notwendigerweise mit einer Verringerung der Kinderzahl pro Frau und damit einem Rückgang der vollständigen Kohortenfertilitätsrate einhergehen muss. Bei einem Rückgang der Periodenfertilitätsrate ist somit nicht erkennbar, ob dies auf einen vorübergehenden Aufschub von Geburten oder auf eine langfristige Reduktion der Kinderzahl zurückzuführen ist. Desgleichen wird aus einem Anstieg der Geburtenrate nicht ersichtlich, inwieweit dies auf einem Nachholen aufgeschobener Geburten oder auf einem langfristigen Anstieg der Kinderzahl pro Frau beruht. Zum anderen bleiben paritätsspezifische Aspekte und damit familienstrukturelle Komponenten der Fertilitätsentwicklung unberücksichtigt. Da die zusammengefasste Geburtenziffer nur altersspezifische Komponenten berücksichtigt, nicht aber die Zahl der Kinder, die eine Frau hat, können aus der Gesamtfertilitätsrate auch keine Rückschlüsse über das Geburtenverhalten von kinderlosen Frauen, Frauen mit einem Kind, Frauen mit zwei Kindern, usw. gezogen werden. Unklar bleibt daher, ob etwa ein Rückgang der Gesamtfertilitätsrate auf einem Anstieg der Kinderlosigkeit oder einem Rückgang an Zweit-, Dritt- oder weiteren Geburten beruht. Veränderungen der Periodenfertilitätsraten geben somit keinen Aufschluss darüber, ob sie auf eine allgemeine Veränderung im Geburtenverhalten aller Frauen oder auf eine Veränderung im Geburtenverhalten einer bestimmten Gruppe von Frauen zurückzuführen sind. Methoden, die periodenspezifische und geburtenspezifische Veränderungen vollständig darstellen, setzen jedoch Datensätze voraus, die genaue Informationen über die Geburtenfolge im Lebensverlauf jeder Frau beinhalten. In den nordischen Ländern stehen mit den Registerdaten solche Datensätze zur Verfügung. Diese Individualdaten umfassen vollständige Informationen über die Geburten aller im jeweiligen Land registrierten Frauen. ${ }^{9}$ Für diesen Bericht haben wir die vollständigen Registerdaten Schwedens, Norwegens und Dänemarks und eine 10\% Stichprobe aus den Registerdaten Finnlands ausgewertet. Die Daten wurden für die vorliegenden Untersuchungen harmonisiert und für eine vergleichende Analyse nutzbar gemacht. Mit Hilfe ereignisanalytischer Methoden können wir daher die alters-, paritäts- und periodenspezifischen Entwicklungen in den nordischen Staaten vergleichend darstellen.

\footnotetext{
${ }^{9}$ Für Deutschland fehlen äquivalente Datensätze. Die amtliche Statistik erfasst die Ordnung der Geburten nur innerhalb einer bestehenden Ehe. Mikrozensus, Panel- und andere Surveydaten, in denen die Geburtenfolge unabhängig vom Familienstand erhoben wird, können zwar partiell diesen Mangel ausgleichen, doch sind auch sie für Untersuchungen der Fertilitätsentwicklung nur mit Einschränkungen geeignet. Der Mikrozensus, dessen Befragungspopulation alle vier Jahre vollständig wechselt, erhebt nur die im Haushalt lebenden Kinder. Dies erschwert Untersuchungen über das Gebärverhalten und damit über Veränderungen im Fertilitätsverhalten über längere Zeiträume. Andere Datenquellen, wie etwa der Familiensurvey, das Sozio-ökonomische Panel oder der Fertility and Family Survey lassen zwar solche Untersuchungen generell zu, erlauben allerdings aufgrund anderer Gegebenheiten, z.B. aufgrund der Stichprobengröße, keine differenzierteren Analysen. Zu einem zusammenfassenden Überblick über die Problematik in Deutschland am Beispiel der Kinderlosigkeit siehe Kreyenfeld 2004a.
} 


\subsection{Die altersspezifische Entwicklung der Familiengründung: Erstgeburtenraten in den nordischen Ländern}

Das unterschiedliche Fertilitätsniveau in den westeuropäischen Ländern wird zu einem großen Teil durch das Geburtenverhalten der über 30-jährigen Frauen bestimmt. Denn in allen europäischen Ländern ist das mittlere Gebäralter von Frauen bei der Geburt ihres ersten Kindes deutlich angestiegen. In den nordischen Ländern lag das Durchschnittsalter aller erstgebärenden Frauen 1980 bei 27 Jahren, 2003 bei 30 Jahren (Council of Europe 2004). Diese Entwicklungen gehen mit einem Rückgang der Erstgeburtenraten ${ }^{10}$ von Frauen der Altersgruppe 15 bis 29 Jahre einher. Wie Grafik 1 veranschaulicht, hat sich die Tendenz zu einer Erstgeburt unter kinderlosen 15- bis 29-jährigen Frauen in den nordischen Ländern seit den 1970er Jahren deutlich verringert. Insbesondere in den 1970er und frühen 1980er Jahren haben sich junge Frauen in den nordischen Ländern wesentlich seltener für eine Mutterschaft entschieden als noch zu Beginn der 1970er Jahre. Im darauf folgenden Jahrzehnt stabilisierte sich die Erstgeburtenrate junger Frauen in Dänemark, Norwegen und Finnland. Seit den1990er Jahre scheint sie jedoch wieder leicht zu sinken. Schweden verzeichnete von Mitte der 1980er bis zu Beginn der 1990er Jahre einen Anstieg der Erstgeburtenrate junger Frauen, danach jedoch einen deutlichen Rückgang. Wie in den 1980er Jahren bleiben in Schweden Ende der 1990er Jahres Frauen unter 30 häufiger kinderlos als in den anderen nordischen Ländern, während Frauen in Norwegen in höherem Ausmaß als in den anderen nordischen Ländern schon in jüngeren Jahren Mutter werden. ${ }^{11}$ Insgesamt betrachtet ist in den nordischen Staaten seit Anfang der 1970er Jahre die Erstgeburtenrate junger Frauen um rund die Hälfte bis zwei Drittel gesunken.

Grafik 1: Standardisierte Erstgeburtenraten kinderloser unter 30-jähriger Frauen in den nordischen Ländern

Anders verlief die Entwicklung der Fertilität kinderloser Frauen im Alter zwischen 30 und 44. Bis Anfang der 1980er Jahre sind in den nordischen Ländern kaum signifikante Änderungen im Gebärverhalten dieser Frauen zu erkennen. Ab Mitte der 1980er Jahre stiegen die Erstgeburtenraten der 30- bis 44-jährigen Frauen in allen nordischen Ländern jedoch deutlich an. In den skandinavischen Staaten hielt dieser Trend im Wesentlichen bis

\footnotetext{
${ }^{10} \mathrm{Da}$ unseren Berechnungen ein ereignisanalytisches Modell zugrunde liegt, sind die Raten statistisch „relative Risiken”. Sie sind standardisiert nach dem Alter der Mutter und - bei Geburten höherer Ordnung - auch nach Geburtenabstand. In den Grafiken sind die Werte relativ zu Schweden im Jahre 1977 angegeben. Aus Gründen der besseren allgemeinen Verständlichkeit verwenden wir statt „(relatives) Risiko einer ersten Geburt“ meistens die Bezeichnung "Erstgeburtenrate”, gelegentlich auch „standardisierte Erstgeburtenrate“. Ähnliches gilt für die Geburtenraten höherer Ordnung.

${ }^{11}$ Nach Skrede (2003) ist die höhere Erstgeburtenrate unter jungen Frauen in Norwegen zum Teil auf die spezifische Unterstützung für allein stehende Mütter in diesem Lande zurückzuführen.
} 
zur Jahrtausendwende. Nur in Schweden entschlossen sich in den späten 1990er Jahren kinderlose Frauen dieser Altersgruppe etwas seltener dazu, ein Kind zu bekommen, so dass der Anstieg der Erstgeburtenrate vorübergehend stagnierte bzw. sogar leicht rückläufig war. Dennoch wurden auch in diesem Zeitraum über 30-jährige kinderlose Frauen häufiger Mutter als in den 1980er Jahren. Dasselbe gilt für Finnland, wo im Gegensatz zu den drei skandinavischen Ländern die Erstgeburtenrate über 30-jähriger Frauen seit den 1990er Jahren stagniert. Finnische Frauen dieser Altersgruppe weisen im Vergleich zu den anderen nordischen Ländern ein insgesamt niedrigeres Risiko einer Erstgeburt auf. $\mathrm{Da}$ die Erstgeburtenrate von Frauen unter 30 in etwa jener der anderen nordischen Länder entspricht, zeigt dies eine höhere Kinderlosigkeit finnischer Frauen an.

Der Rückgang der Erstgeburtenraten kinderloser unter 30-jähriger Frauen in den 1970er und frühen 1980er Jahren wurde in den nordischen Ländern durch einen Anstieg der Erstgeburtenraten kinderloser über 30-jähriger Frauen ab Mitte der 1980er Jahre überwiegend kompensiert. Diese Entwicklung spiegelt eine grundlegende Änderung im Reproduktionsverhalten kinderloser Frauen wider. Immer mehr Frauen in den nordischen Ländern bleiben in jüngeren Jahren (bis 30 ) kinderlos und bekommen ihr erstes Kind erst in einem späteren Lebensabschnitt (ab 30).

Grafik 2: Standardisierte Erstgeburtenraten kinderloser über 30-jähriger Frauen in den nordischen Ländern

In keinem der nordischen Länder scheint der Aufschub der Familiengründung unter jüngeren Frauen zu einem bedeutenden Anstieg der Kinderlosigkeit geführt zu haben. Von den 1945-49 geborenen Frauen blieb in Finnland jede sechste (17\%), in den skandinavischen Ländern rund jede zehnte Frau kinderlos (Norwegen 9\%, Dänemark 10\%, Schweden 11\%). In Dänemark, Norwegen und Schweden stieg der Anteil der kinderlosen Frauen bis zu den Geburtenkohorten 1955-59 um 2 bis 4 Prozentpunkte an, in Finnland blieb der Anteil der permanent Kinderlosen gleich. Von den 1955-59 geborenen Frauen hatten somit zwischen $12 \%$ (Norwegen) und 17\% (Finnland) keine eigenen Kinder.

Diese Entwicklung unterscheidet sich deutlich von jener in Westdeutschland, wo insbesondere die ab den 1950er Jahren geborenen Frauen sich wesentlich häufiger gegen eine Mutterschaft entschieden. Von den zwischen 1945 und 1949 geborenen westdeutschen Frauen blieben zwischen 12\% und 14\% kinderlos; von den 1955-59 geborenen Frauen sind es zwischen 22\% (Geburtenkohorte 1955) und 26\% (Geburtenkohorte 1959) (New Cronos 2001; eigene Berechnungen). Auf die Gesamtfertilitätsraten bezogen bedeutet dies, dass die Entwicklung der Gesamtfertilität in Deutschland und in den nordischen Ländern auf teilweise unterschiedlichen Gründen beruht. In Deutschland scheinen der Rückgang der Gesamtfertilität und der Anstieg des Gebäralters mit einer Zunahme der Kinderlosigkeit 
einherzugehen, wenngleich noch immer die überwiegende Mehrzahl von Frauen im Laufe ihres Lebens Mütter werden. ${ }^{12}$ In den nordischen Ländern sind Rückgang und Anstieg der Gesamtfertilitätsrate seit den 1970er Jahren dagegen auf einen Aufschub der Familiengründung unter jüngeren Frauen und auf eine Entscheidung zur Mutterschaft in einem höheren Lebensalter zurückzuführen.

\subsection{Die partitätsspezifische Entwicklung der Fertilität von Müttern in den nordischen Ländern}

Demografische Studien haben wiederholt nachgewiesen, dass Frauen, die erst spät ihr erstes Kind bekommen, im Schnitt weniger Kinder haben als Frauen, die schon in jungen Jahren Mütter werden. Auch für die nordischen Länder gilt dieser Zusammenhang. Dennoch bedarf die Annahme einer gewissen Modifikation. Bereits auf der Aggregatebene zeigen Kohortendaten, dass sich trotz Anstiegs des Alters von Frauen bei ihrer Erstgeburt der Anteil von Müttern mit einem, zwei, drei und mehr Kindern in den nordischen Staaten nur geringfügig geändert hat. Lediglich in Norwegen brachten Frauen jüngerer Geburtskohorten im Verlaufe ihres Lebens deutlich seltener drei und mehr Kinder zur Welt als Frauen älterer Geburtskohorten (Anteil der Frauen des Geburtsjahrganges 1960 mit drei und mehr Kindern: 34\%; des Geburtsjahrganges 1940: 47\%; New Cronos 2001). In Schweden und Dänemark reduzierte sich der Anteil von Frauen mit drei und mehr Kindern im Kohortenvergleich (1940, 1960) um 1 bis 3 Prozentpunkte, in Finnland stieg der Anteil dieser Frauen um 2 Prozentpunkte. ${ }^{13}$ Die vollendete Zahl der Kinder, so deuten diese Vergleiche an, wird nicht allein durch das Alter der Frau bei der Erstgeburt beeinflusst, sondern auch durch den Abstand zwischen den Geburten.

Grafik 3 zeigt die Zweitgeburtenraten für die nordischen Länder seit den 1970er Jahren, wobei das Alter der Mutter und das Alter des ersten Kindes (also der Geburtenabstand zum zweiten Kind) in die Berechnungen der Raten einbezogen wurden. In der ersten Hälfte der 1970er Jahre waren die Zweitgeburtenraten in allen nordischen Ländern, ausgenommen Finnland, rückläufig. Das bedeutet, dass Mütter mit einem Kind in Schweden, Norwegen und Dänemark in diesem Zeitraum tendentiell weniger dazu neigten, ein zweites Kind zu bekommen oder eine zweite Geburt aufschoben. In Dänemark dauerte der Rückgang noch bis 1983, in Schweden und Norwegen trat bereits 1977 eine Trendwende ein. Alle nordischen Länder verzeichnen während der 1980er Jahre einen Anstieg ihrer Zweitgeburtenraten. Am Ausgeprägtesten war dieser in Schweden, Finnland und Dänemark.

\footnotetext{
${ }^{12}$ Der Rückgang der Periodenfertilitätsrate in Deutschland ist daher auch nicht allein der zunehmenden Kinderlosigkeit zuzuschreiben; Tempoeffekte und der Rückgang an Geburten höherer Ordnung spielen ebenfalls eine Rolle.

${ }^{13}$ Vergleiche mit Deutschland sind nur eingeschränkt möglich, weil in Deutschland die Rangordnung der Kinder nur in aufrechter Ehe erhoben wird. Deutschland verzeichnet im Kohortenvergleich einen Rückgang der Zahl der Kinder in allen Gruppen (Geburtskohorten 1940 - 1959: Frauen mit einem Kind: -3\%; Frauen mit zwei Kindern: - 6\%; Frauen mit drei und mehr Kindern: -1\%).
} 
In Dänemark dauerte der Anstieg bis zur Gegenwart (2001) fort. In Finnland und Norwegen stagniert die Zweitgeburtenrate im Wesentlichen seit Anfang der 1990er Jahre. Seit der Trendwende ist die standardisierte Zweitgeburtenrate in Dänemark und Finnland um rund 40 Prozentpunkte und in Norwegen um etwa 15 Prozentpunkte gestiegen. Weit weniger stabil verlief die Entwicklung in Schweden. Dort stieg die Zweitgeburtenrate von Ende der 1970er Jahre bis zu Beginn der 1990er Jahre um rund 65 Prozentpunkte an, fiel jedoch zwischen 1991 und 1997 um ein Viertel auf etwa das Niveau von 1985 zurück (Hoem 1993; Andersson 1999). Trotz dieses Rückgangs weist Schweden noch immer die höchste Zweitgeburtenrate der nordischen Länder auf. Von den nationalen Besonderheiten in der Entwicklung abgesehen, weisen zu Beginn des 21. Jahrhunderts alle nordischen Länder höhere standardisierte Zweitgeburtenraten auf als zu Beginn der 1980er Jahre (Grafik 3).

Grafik 3. Standardisierte Zweitgeburtenraten von Müttern mit einem Kind in den nordischen Ländern

Bei den dritten Geburten ist die Entwicklung zum Teil ähnlich wie bei den Zweitgeburten, doch die relativen Änderungen sind etwas ausgeprägter als bei Zweitgeburten. In allen Ländern (ausgenommen Finnland) sinkt die Rate in den 1970er Jahren, steigt in den 1980er Jahre jedoch ebenfalls an. In allen vier Ländern erfolgt dieser Anstieg relativ konstant. Seit den frühen 1990er Jahren verzeichnen Norwegen, Finnland und Dänemark keinen weiteren Anstieg der Drittgeburtenrate. Wie bei den zweiten Geburten fiel in Schweden auch die Rate der dritten Geburten zwischen 1990 und 1997 erheblich. Schweden, das während der 1980er Jahre das Land mit der höchsten Drittgeburtenrate unter den nordischen Ländern war, weist seit Mitte der 1990er Jahre die niedrigste Drittgeburtenrate der nordischen Staaten auf. (Grafik 4$)^{14}$

Grafik 4: Standardisierte Drittgeburtenrate von Müttern mit zwei Kindern in den nordischen Ländern

\footnotetext{
${ }^{14}$ Der Trend der Viertgeburtenraten verläuft für Dänemark, Norwegen und Schweden im Wesentlichen analog der Entwicklung der Drittgeburtenraten: Nach einem Rückgang der Raten in den 1970er Jahren, verzeichnen diese Länder einen Anstieg der relativen Raten in den 1980er Jahre. In Dänemark und Norwegen bleiben die Viertgeburtenraten danach relativ konstant; in Schweden steigt die Rate nach einem Rückgang zwischen 1990 und 1997 wieder an. Im Unterschied zu den Zweit- und Drittgeburtenraten liegt jedoch die Viertgeburtenrate in Finnland seit den 1970er Jahren etwas über den Raten der anderen nordischen Länder. $\mathrm{Da}$ - aus der Kohortenperspektive betrachtet - jedoch nur ein geringer Prozentsatz aller Frauen in den nordischen Ländern ein viertes Kind bekommt, stellen wir die Viertgeburtenraten hier nicht gesondert dar.
} 
Betrachtet man die Entwicklung aller Paritäten, so zeigt sich in der Tendenz ein relativ einheitliches Bild. In allen nordischen Ländern sind die Raten zu Beginn des 21. Jahrhunderts höher als in den 1970er Jahren. Der Anstieg setzte bereits in den 1980er Jahren ein und verlief in Dänemark, Norwegen und Finnland relativ stabil. In Schweden hingegen unterlag die Entwicklung markanten Schwankungen mit einem starken Anstieg der Zweit- (bzw. Dritt- und Viert-)geburtenrate in den 1980er Jahren, einem deutlichen Rückgang in der ersten Hälfte der 1990er Jahren und einem nachfolgenden Wiederanstieg.

Angesichts des steigenden Alters von Frauen bei der Erstgeburt (siehe Grafik 1 und Grafik 2) drängt sich die Frage nach dem Geburtenverhalten von Müttern bestimmter Altersgruppen auf. Grafik 5 und Grafik 6 zeigen die relativen Zweit- und Drittgeburtenraten nach dem Alter der Mutter. Sie veranschaulichen, in welchem Maße Mütter einer bestimmten Altersgruppe, die bereits ein bzw. zwei Kinder haben, dazu tendieren, ein weiteres Kind zu bekommen. In Bezug auf dritte Geburten ergibt sich ein relativ einheitliches Bild: In allen nordischen Ländern sinkt mit steigendem Alter die Neigung von Müttern mit zwei Kindern, ein drittes zu haben (Grafik 6). Anders die Verteilung der Zweitgeburtenrate. Wie aus Grafik 5 ersichtlich, fällt in allen Ländern das relative Risiko einer zweiten Geburt erst für Mütter ab Mitte 30 deutlich ab. Bei diesen Müttern kann davon ausgegangen werden, dass zumindest ein Teil von innen aufgrund abnehmender Fruchtbarkeit kein weiteres Kind mehr bekam, obwohl sie sich noch ein Kind wünschten. Unter 34-jährige Mütter (mit einem Kind) tendieren in Schweden etwas mehr dazu, ein zweites Kind zu haben als Mütter in den anderen nordischen Ländern. Doch insgesamt betrachtet, gibt es in den nordischen Ländern nur geringe Unterschiede in der Verteilung der Rate der Zweitgeburten über die Altersgruppen hinweg.

Grafik 5: Altersspezifische standardisierte Zweitgeburtenraten von Müttern mit einem Kind in den nordischen Ländern

Grafik 6: Altersspezifische standardisierte Drittgeburtenraten von Müttern mit zwei Kindern in den nordischen Ländern

Betrachtet man nur die demografische Seite, so lassen sich die Differenzen in den paritätsspezifischen Raten der nordischen Länder zum Teil darauf zurückführen, in welchem zeitlichen Abstand von einer vorangegangenen Geburt Frauen ihr weiteres Kind bekommen. In dieser Hinsicht bestehen sowohl zwischen den einzelnen Ländern als auch über den Zeitverlauf hinweg bemerkenswerte Unterschiede. In Dänemark und Norwegen bekommen Frauen ihr zweites Kind meist zwei bis vier Jahre nach dem ersten, wobei es in Norwegen in der letzten Dekade eine leichte Tendenz hin zu einer Verkürzung des Geburtenabstandes auf zwei bis drei Jahre gibt. Anders die Situation in Schweden und Finnland. In Finnland 
bevorzugten Frauen schon 1980 einen zeitlichen Abstand von zwei bis drei Jahren zwischen der ersten und zweiten Geburt; diese Präferenz hat sich bis 2000 kontinuierlich verstärkt. In Schweden neigten Frauen, ähnlich wie in Dänemark und Norwegen, 1980 noch dazu, ihr zweites Kind rund zwei bis vier Jahre nach dem ersten zu bekommen. 1990 wählten sie jedoch bereits überwiegend einen Geburtenabstand von zwei bis drei Jahren. Die Veränderung im Geburtenverhalten war nicht kontinuierlich, sondern konzentrierte sich im Wesentlichen auf die Jahre 1980 und 1986. Wie im folgenden noch erörtert wird, verdankt sich diese Entwicklung der Einführung einer familienpolitischen Leistung in Schweden, die jene Frauen unterstützt, die ihr zweites oder weiteres Kind innerhalb einer bestimmten Frist nach dem vorangegangenen bekommen (Hoem 1990; 1993; Andersson 1999; 2004; Andersson/Hoem/Duvander 2006). Die Verkürzung des Geburtenabstandes hat in den 1980er Jahren in Schweden auch entscheidend zum signifikanten Anstieg der Zweit- (und Dritt-)geburten beigetragen (Andersson 2004).

Zusammenfassend betrachtet zeichnen unsere Analysen ein gegenüber den bloßen Aggregatdaten deutlich differenzierteres Bild. Zum einen zeigt sich, dass der Anstieg der Gesamtfertilitätsraten in den nordischen Ländern und ihr im europäischen Vergleich relativ hohes Niveau auf zwei Faktoren zurückzuführen ist: erstens auf den Anstieg der Erstgeburtenraten unter den über 30-jährigen Frauen, und zweitens auf einen Anstieg der Raten zweiter und dritter Geburten. Diese Entwicklungen sind in allen nordischen Ländern zu beobachten. Hinsichtlich dieser beiden Entwicklungen scheinen die nordischen Länder tatsächlich ein einheitliches „demografisches Regime“ zu bilden, wie von der Forschung immer wieder konstatiert (Andersson 2004; Vikat 2004). Zum anderen bestehen jedoch auch Unterschiede zwischen den Ländern, insbesondere in Bezug auf das Niveau der Raten, ihre periodenspezifischen Variationen und in Bezug auf die Geburtenabstände. Wie wir im Abschnitt 4 zeigen werden, stehen beide, die demografischen Gemeinsamkeiten ebenso wie die nationalstaatlichen Besonderheiten der demografischen Entwicklung in einem Zusammenhang mit der Ausgestaltung des Wohlfahrtsstaates und der Familien- und Geschlechterpolitik in den nordischen Ländern.

\section{Geburtenentwicklung und Bildung in den nordischen Ländern}

Der Rückgang der Fertilität in Europa und die unterschiedliche Entwicklung der Fertilität in den einzelnen Ländern werden häufig auf die steigende Bildungsbeteiligung und Erwerbstätigkeit von Frauen zurückgeführt. Steigende Ausbildungsinvestitionen und Erwerbstätigkeit, so die Argumentation, erhöhten die Opportunitätskosten und führten daher zu einem Verzicht auf Kinder oder zu einer Reduktion der Kinderzahl. Demografische Untersuchungen über den Zusammenhang von Bildung und Fertilität scheinen diese Annahme zu bestätigen: Frauen mit einem höheren Ausbildungsgrad bleiben häufiger 
kinderlos und haben im Verlaufe ihres Lebens weniger Kinder als Frauen mit einem niedrigeren Ausbildungsgrad. Forschungen zu den nordischen Ländern, aber auch zu Deutschland zeigen jedoch, dass dieser Zusammenhang keineswegs universell gültig ist. So weist etwa B. Hoem (1993) darauf hin, dass in Schweden schon in den 1980er Jahren hoch qualifizierte Frauen nur geringfügig häufiger kinderlos blieben als Frauen mit niedrigeren Bildungsabschlüssen (B. Hoem 1993: 116). Vergleichende Studien zu Bildung und Fertilität zwischen Ost- und Westdeutschland stellen einen generell negativen Zusammenhang zwischen Bildungshöhe und Kinderlosigkeit ebenfalls in Frage. Kreyenfeld (2004b), Grünheid 2004 und Wirth/Dümmler (2004) zeigen, dass die hohe Kinderlosigkeit unter Hochschul- und Fachhochschulabsolventinnen in Deutschland primär ein Phänomen Westdeutschlands ist. ${ }^{15}$ Neuere Kohortenuntersuchungen konstatieren sogar einen Anstieg der Kinderlosigkeit unter hoch qualifizierten Frauen in Westdeutschland, die mit einem Anstieg der Unehelichkeit in dieser Gruppe von Frauen korrespondiert (Wirth/Dümmler 2004). Eine analoge Entwicklung ist in den nordischen Ländern nicht feststellbar (für Schweden siehe: Hoem/Neyer/Andersson 2006a; für Norwegen: Lappegård/Rønsen 2005).

Alle Analysen verweisen jedoch auch darauf, dass die Fertilitätsunterschiede zwischen den Bildungsgruppen zu einem erheblichen Teil auf die unterschiedliche Dauer der Ausbildung zurückzuführen sind (Blossfeld/Huinink 1991; B. Hoem 1993; Wirth/Dümmler 2004). Frauen in Ausbildung zeigen in allen Ländern eine deutlich geringere Neigung, ein Kind zur Welt zu bringen, als Frauen, die das Bildungssystem verlassen haben. Die geringere Fertilität von Frauen mit höherer Ausbildung ist daher partiell auf den Aufschub von Geburten unter Frauen mit längerer Ausbildungsdauer zurückzuführen. Sofern hoch qualifizierte Frauen nach dem Verlassen des Bildungssystems Mütter werden, weisen sie im Allgemeinen eine höhere oder äquivalente Fertilitätsrate (Zweit- und Drittgeburten) auf als Frauen mit einem geringeren Ausbildungsgrad (Blossfeld/Huinink 1991; Kravdal 2001; B. Hoem 1993; Hoem/Prskawetz/Neyer 2001). Die für die Fertilitätsentwicklung zentrale Frage ist somit, ob Frauen auch nach einer länger dauernden Ausbildung eine Mutterschaft wagen oder gänzlich darauf verzichten. In dieser Hinsicht scheinen zwischen den nordischen Ländern und Deutschland deutliche Unterschiede zu bestehen.

Huinink (2002) hat für Westdeutschland gezeigt, dass die Fertilität unter hochqualifizierten Frauen bi-modal (also U-förmig) verteilt ist. Frauen mit einem hohen Ausbildungsgrad haben entweder keine oder zwei Kinder. Eine solche Polarisierung lässt sich für andere Bildungsgruppen nicht nachweisen. Grünheid (2004) folgert aus einem Kohortenvergleich, dass jüngere Akademikerinnen in Westdeutschland stärker zu einem bzw. zwei Kindern neigen und die Kinderlosigkeit zurückgeht. Allerdings stellt sie bei westdeutschen Frauen mit mittlerem Bildungsabschluss oder einer Fachausbildung eine

15 Wie Neyer und Hoem (2006, im Erscheinen) zeigen, weisen auch österreichische Hochschulabsolventinnen eine deutlich höhere Kinderlosigkeit auf als Frauen mit geringeren Bildungsabschlüssen. Dies unterstützt die Annahme, dass die institutionellen Rahmenbedingungen einen wesentlichen Faktor in bezug auf bildungsdifferentielle bzw. bildungsäquivalente Fertilitätsraten bilden (siehe unten). 
zunehmende Polarisierung zwischen Frauen, die kinderlos bleiben und Frauen, die zwei (oder mehr) Kinder haben, fest. Bei ostdeutschen Frauen sind diese Polarisierungen nicht feststellbar. Die Verschiebungen in der Verteilung der Geburten scheinen im Zusammenhang mit der Entwicklung des Familienstandes zu stehen. Unverheiratete westdeutsche Frauen bleiben eher kinderlos, während der Familienstatus für ostdeutsche Frauen kaum einen Einfluss auf ihre Entscheidung zur Mutterschaft hat. Verheiratete westdeutsche Frauen tendieren zu zwei Kindern, ostdeutsche Frauen zu einem oder zwei Kindern (Grünheid 2004: 41). Bemerkenswert ist in diesem Zusammenhang, dass die Fertilität in Westdeutschland nicht nur durch den Bildungsgrad der Frau, sondern mehr noch durch den Bildungsgrad des Partners bestimmt wird (Kreyenfeld 2002). ${ }^{16}$

In den nordischen Ländern weichen Entwicklung und Verteilung der Fertilität nach Ausbildungsebene deutlich von jener in Deutschland ab. Schon in den 1980er Jahren erwiesen sich Fertilität und Familienstand in den nordischen Ländern als weitgehend entkoppelt. Ob ein Paar unverheiratet war, hatte nur einen geringen Einfluss auf eine potentielle oder weitere Elternschaft. Studien zu Norwegen (Kravdal 1992; 1996; 2001) und Schweden (Hoem/Hoem 1989; B. Hoem 1993) zeigen, dass die Unterschiede in der Fertilität zwischen Frauen unterschiedlicher Ausbildungsgruppen zudem geringer geworden sind. Die Wende von höherer Kinderlosigkeit und geringeren Fertilitätsraten unter höher qualifizierten Frauen zu einer Angleichung ihres Fertilitätsverhaltens an das anderer Ausbildungsgruppen beginnt mit den Geburtskohorten der frühen 1950er Jahre (Kravdal 2001; Lappegård/Rønsen 2005). Diese Geburtskohorten konnten auch als erste voll von der Expansion des Bildungswesen, des (weiblichen) Arbeitsmarktes, der sozialen Dienstleistungen und den politischen Anstrengungen zur Verringerung der Geschlechterdifferenzen im Ausbildungssystem und am Arbeitsmarkt profitierten. Gerade angesichts dieser Entwicklungen stellt sich die Frage nach den Zusammenhängen zwischen Bildung und Geburtenverhalten.

Neuere Untersuchungen auf der Basis von Registerdaten zeigen, dass durch die Konzentration der Forschung auf das Ausbildungsniveau wesentliche Aspekte des Zusammenhangs von Bildung und Fertilität verdeckt bleiben. Auswertungen von schwedischen und norwegischen Registerdaten offenbaren, dass die Ausbildungsrichtung ein entscheidender Faktor in Bezug auf Mutterschaft und Kinderzahl darstellt (Hoem/Neyer/Andersson 2006a; 2006b; Lappegård 2002; Lappegård/Rønsen 2005). Zwar bestätigen auch diese Untersuchungen, dass Frauen mit einer höheren Ausbildung häufiger kinderlos bleiben und im Verlaufe ihres Lebens eine geringere Zahl von Kindern zur Welt bringen als Frauen mit einem niedrigeren Bildungsgrad. Doch diese Feststellung beschränkt sich im Wesentlichen auf Frauen derselben Ausbildungsrichtung (siehe Grafik 7 und Grafik 8). Unabhängig von der Höhe des Bildungsabschlusses weisen Frauen, die einen Bildungsabschluss für den Unterrichtsbereich oder den Gesundheitsbereich haben, eine deutlich niedrigere Kinderlosigkeit und eine deutlich höhere Zahl an Kindern (vollständige

\footnotetext{
${ }^{16}$ Dasselbe gilt für Österreich. Siehe Hoem/Prskawetz/Neyer 2001.
} 
Fertilität) auf als Frauen, die eine Ausbildung im Verwaltungsbereich, in den Sozialwissenschaften, im Wirtschaftsbereich, aber auch in persönlichen Diensten haben. Insgesamt betrachtet hat die Ausbildungsrichtung einen stärkeren Effekt auf Kinderlosigkeit und Kinderzahl als die Ausbildungshöhe (Hoem/Neyer/Andersson 2006a; 2006b).

Für diese Wirkungen scheinen mehrere miteinander verschränkte Faktoren ausschlaggebend. Auf der individuellen Ebene können Selektions- und Adaptionseffekte zum Tragen kommen. Frauen, die (einmal) Kinder haben möchten, tendieren möglicherweise dazu, Ausbildungsrichtungen zu wählen, die sie für einen Beruf qualifizieren, in dem Elternschaft und Berufstätigkeit leichter vereinbar erscheinen. Frauen, die eine Ausbildung für einen Beruf wählen, in dem sich - praktisch oder normativ - eine Vereinbarkeit von Elternschaft und Berufstätigkeit nur als beschränkt möglich erweist, reduzieren unter Umständen die Zahl ihrer Kinder, verzichten gänzlich auf sie oder, sofern das Ausbildungssystem es zulässt, qualifizieren sich für einen anderen Beruf, in dem auch Mutterschaft realisierbar ist. Diese Aspekte weisen auf die Bedeutung von strukturellen Bedingungen im Bildungssystem und am Arbeitsmarkt hin. Die Flexibilität des Bildungssystems, das, wie im Falle Schwedens, Ein- und Ausstiege, Re- und Neuqualifikationen über den Lebensverlauf hinweg erlaubt, die Geschlechterstrukturen im Ausbildungs- und im Berufsbereich, die Erwerbsmöglichkeiten, die Arbeitsbedingungen und die Einkommenssituation von Frauen scheinen einen wesentlichen Einfluss auf ihre Entscheidung für oder gegen eine Mutterschaft zu haben. Die Möglichkeit, Mutterschaft und Bildung zumindest sequenziell zu verbinden und durch Weiterbildung, Umschulung oder Nachschulung zusätzliche oder neue Qualifikationen zu erwerben, scheinen partiell zu der kaum höheren Kinderlosigkeit bzw. geringeren Kinderzahl unter hoch qualifizierten Frauen in den einzelnen Ausbildungsbereichen zu führen. Dazu trägt auch eine Familien-, Wohlfahrtsstaat- und Geschlechterpolitik bei, die Erwerbsbeteiligung und Bildungspartizipation durch monetäre und dienstleistungsorientierte Angebote unterstützt. Frauen mit einer Ausbildung, die für Berufe mit wenig stabilen Erwerbsaussichten, mit zeitlich belastenden Arbeitsbedingungen oder mit einem - relativ zum Ausbildungsgrad - geringen Einkommen qualifiziert, scheinen eher kinderlos zu bleiben bzw. weniger Kinder zu haben, als Frauen, deren Ausbildung mit der Aussicht auf gesicherte, kontinuierliche und gut (oder zumindest bildungsadäquat) bezahlte Berufstätigkeit verbunden ist. Insgesamt betrachtet verweisen die Analysen, auf die Notwendigkeit, Strukturen des Bildungssystems, des Arbeitsmarktes, Familienpolitik und Geschlechterpolitik und deren Zusammenhänge zur Familiengründung näher zu beleuchten (zu den Details siehe Hoem/Neyer/Andersson 2006a; 2006b).

Grafik 7: Kinderlosigkeit nach Ausbildungsrichtung und Ausbildungsgrad: Schwedische Frauen der Geburtenjährgänge 1955-59. 
Grafik 8: Vollständige Fertilität, Ausbildungsrichtung und Ausbildungsgrad: Schwedische Frauen der Geburtenjahrgänge 1955-59.

\section{Fertilität, Familienpolitik und Wohlfahrtsstaat in den nordischen Ländern}

\subsection{Wohlfahrtsstaat und Familienpolitik: Deutschland und die nordischen Länder}

In der vergleichenden Wohlfahrtsstaatsforschung werden die nordischen Länder als universalistische Wohlfahrtsstaaten angesehen; Deutschland dagegen wird dem konservativfamilialistischen Wohlfahrtsstaatsregime zugerechnet (Esping-Andersen 1990; 1999; Lewis 1992; Langan/Ostner 1991; Sainsbury 1999a). Kennzeichnend für die deutsche Wohlfahrtsstaatspolitik sind ihre Berufs- und Ehezentriertheit, ihre Ausrichtung auf den Erhalt von Statusunterschieden zwischen verschiedenen Bevölkerungsgruppen und ihre Orientierung an einem familienbezogenen Subsidiaritätsprinzip. Nur in vergleichsweise geringem Maße orientiert sich die wohlfahrtsstaatliche Politik an einem universellen Gleichheitsprinzip. Eheliche Familienformen mit einer geschlechtsspezifischen Arbeitsteilung zwischen den Ehepartnern werden im deutschen Wohlfahrtsstaatssystem (aber auch in anderen Bereichen, wie etwa dem Steuerrecht) gegenüber allen anderen Formen von Partnerschaft, Elternschaft und innerfamiliärer Arbeitsteilung privilegiert. Dementsprechend unterstützen Familien- und Sozialpolitik auch nicht den Erhalt der Erwerbstätigkeit von Frauen mit (kleinen) Kindern, sondern begünstigen den Erwerbsausstieg oder die Erwerbsreduktion von Müttern. Dem Subsidiaritätsprinzip folgend kommt der Familie (i.e. der ehelichen und nicht-ehelichen Partnerschaft ebenso wie den Generationenbeziehungen) in der Wohlfahrtsproduktion eine zentrale Bedeutung zu. Soziale Transferleistungen werden in vielen Fällen nicht auf der Basis von individuellen Rechtsansprüchen gewährt, sondern sind an Familienstatus und Familieneinkommen rückgebunden. Gesellschaftlich notwendige Betreuungsaufgaben, wie die Betreuung von Kindern oder älteren pflegebedürftigen Personen, werden vorwiegend als Aufgaben der Familie betrachtet und sozialstaatliche

Dienstleistungen als familienergänzende oder familienunterstützende Maßnahmen angesehen.

Diese Orientierungen spiegeln sich auch in den geburten- und betreuungsbezogenen Maßnahmen der Familienpolitik, bei der Elternzeit, dem Erziehungsgeld und dem Angebot an Kinderbetreuungseinrichtungen, wider. Deutschland gewährt bislang Müttern (und Vätern) nach der Geburt eines Kindes eine im internationalen Vergleich relativ lange Elternzeit (bis zum dritten Geburtstag des Kindes), allerdings mit eingeschränkter Flexibilität hinsichtlich der Gestaltung der Elternzeit (Regelung der Inanspruchnahme; Recht auf Teilzeitarbeit). Unabhängig von einer vorangegangenen Erwerbstätigkeit wird allen Müttern ein Erziehungsgeld gewährt, jedoch nur max. bis zum zweiten Geburtstag des Kindes. Das 
Erziehungsgeld besteht im Wesentlichen aus einem einheitlichen, relativ geringen Regelsatz, bei dessen Berechnung ein eigenes Einkommen oder ein Einkommen des Partners/der Partnerin berücksichtigt wird (zu den Details siehe: Bundesministerium 2002). Trotz einiger Modifikationen in den letzten Jahren ermöglichen die Elternzeitregelungen somit nur in sehr begrenztem Maße die Vereinbarkeit von Berufstätigkeit und Kinderbetreuung während der ersten Lebensjahre des Kindes, gewähren aber auch keine hinreichende monetäre Absicherung für Zeiten, in denen Elternzeit in Anspruch genommen wird (zu den ab 2007 geplanten Änderungen siehe unten).

Die Verfügbarkeit von Kinderbetreuungseinrichtungen variiert in Deutschland beträchtlich. In Westdeutschland ist die Quote der verfügbaren Krippenplätze für unter 3jährige Kinder und der verfügbaren Hortplätze für schulpflichtige Kinder in den letzten dreißig Jahren mit rund $3 \%$ (Krippenplätze) und $6 \%$ (Hortplätze) ${ }^{17}$ unverändert niedrig geblieben (Hank/Kreyenfeld/Spieß 2004). In Ostdeutschland ist trotz eines Rückgangs der Krippenplätze nach der Wiedervereinigung die Betreuungssituation erheblich besser als in Westdeutschland. Die Versorgungsquote im Krippenbereich lag 2002 bei 37\%; jene im Hortbereich bei $59 \%$ (Hank/Kreyenfeld/Speiß 2004). Der Kindergartenbereich (für Kinder zwischen 3 und 6 Jahren) wurde in Westdeutschland zwar in den letzten Jahrzehnten deutlich erweitert und damit eine Annäherung an die Versorgungsquote in Ostdeutschland erreicht. Allerdings bieten in Westdeutschland die Kindergärten meist nur Halbtagsbetreuung an, in Ostdeutschland dagegen Ganztagsbetreuung (Deutsches Jugendinstitut 2002).

Deutlich anders ausgerichtet als in Deutschland sind die Wohlfahrtsstaats- und die Familienpolitik in den nordischen Ländern. Sie zeichnen sich durch einen hohen Grad an Arbeitsmarktzentrierung, Gleichheitsorientierung, Individualisierung und der Ausrichtung am Prinzip sozialer Staatsbürgerschaft (d.h. am Prinzip universeller, individueller Rechtsansprüche) aus (Bergqvist et al. 1999; Sainsbury 1999a; 1999b; Gornick 1999; Esping-Andersen 2002; Meyers/Gornick/Ross 1999; Anttonen/Sipilä 1996). Die Sozialpolitik der nordischen Länder stellt auf die Integration aller erwerbsfähigen Frauen und Männer in den Arbeitsmarkt ab. Individuelle soziale Leistungen im Falle von Arbeitslosigkeit oder Elternzeit sind eng an eine vorangegangene Erwerbstätigkeit gebunden. Sie werden in einer Höhe gewährt, die den Erhalt des Lebensstandards auch während der Arbeitsmarktabsenz sicherstellt. Personen ohne vorangegangene Erwerbstätigkeit erhalten einen Grundsicherungsbetrag. Soziale Leistungen werden auf der Basis individueller Rechtsansprüche, die sich aus dem Aufenthalt im jeweiligen Land ableiten, gewährt und sind im Allgemeinen nicht an den Familienstand gekoppelt. Die Sozial- und Familienpolitik ist darauf ausgerichtet, Gleichheit zwischen verschiedenen Bevölkerungsgruppen herzustellen, wie etwa zwischen den Geschlechtern oder zwischen Kindern unterschiedlicher Herkunft. Die Arbeitsmarkt- und Gleichheitsprämissen haben schon seit den 1970er Jahren die Entwicklung der Sozial- und Familienpolitik in den nordischen Ländern bestimmt. Dementsprechend sind

\footnotetext{
${ }^{17}$ Die Prozentsätze geben den Anteil der verfügbaren Plätze an der Zahl der unter 3-jährigen Kinder (Krippe) bzw. der 6- bis 10-jährigen Kinder (Horte) an.
} 
arbeitsmarktbezogene Elternurlaubsregelungen und ein ausgebautes Netz an Kinderbetreuungseinrichtungen für Kinder aller Altersgruppen kennzeichnend für die nordischen Länder.

\section{(a) Elternzeitregelungen in den nordischen Ländern}

Wie in Deutschland wurden die Elternzeitregelungen in den nordischen Ländern zunächst eingeführt, um Müttern/Eltern die Betreuung ihres Kindes in der ersten Zeit nach der Geburt zu ermöglichen. Finnland und Schweden bauten ihre Elternzeit während der 1970er Jahre deutlich aus; Dänemark und Norwegen folgten in den 1980er Jahren (Für einen Überblick über die Entwicklung siehe Rønsen/Sundström 2002; Bergqvist 1999; Hiilamo 2002; Knudsen 2002; Kolbe 2002; Rostgaard/Christoffersen/Weise 1999). Bereits in den 1970er Jahren war die Politik vom Bestreben getragen, Müttern die Erwerbstätigkeit zu ermöglichen, ihren Lebensunterhalt auch während einer Elternzeit zu sichern und Geschlechterdifferenzen am Arbeitsmarkt und in der Kinderbetreuung zu minimieren. Dem entsprechend erhöhten Schweden, Norwegen und Finnland schon in den 1970er bzw. Anfang der 1980er Jahre ihre ursprünglich niedrigen Einkommensersatzleistungen während der Elternzeit auf $90 \%$ (Schweden: 1974), 100\% (Norwegen:1978) und 80\% (Finnland:1982) des vorangegangenen Einkommens (Rønsen/Sundström 2002). ${ }^{18}$ Obwohl Schweden und Finnland in der Rezessionsphase der 1990er Jahre den prozentuellen Einkommensersatz reduzierten, behielten beide Länder mit $75 \%$ (Schweden) bzw. durchschnittlich $71 \%$ (Finnland) ${ }^{19}$ relativ hohe Kompensationen für den Einkommensverlust für Eltern während der Elternzeit bei (Bergqvist 1999, S. 126). Schweden erhöhte sogar nach zwei Jahren 1998 das Elterngeld von $75 \%$ auf $80 \%$.

Die schwedische Elterngeldregelung besitzt seit den 1980er Jahren eine geburtenbezogene Besonderheit. Eltern, die ihr zweites oder weiteres Kind innerhalb von 30 Monaten (seit 1986; davor ab 198024 Monate) nach dem vorangegangenen Kind bekommen, können Elterngeld auf der Basis des Einkommens erhalten, das sie vor dem vorangegangen Kind hatten, sodass eine Reduktion des Einkommens (etwa durch Teilzeitbeschäftigung, Elternzeit oder Erwerbsverzicht nach dem vorangegangenen Kind) sich nicht auf die Höhe des Elterngelds auswirkt (Hoem 1990; 1993). In Dänemark wurde Elterngeld bei seiner Einführung (1984) in Relation zum Arbeitslosengeld bezahlt; kollektive Tarifverträge sehen zu einem großen Teil aber vollen Lohnausgleich vor (Rostgaard/Christophersen/Weise 1999; Knudsen 2002; Etherington 1998; Bergqvist 1999).

Ebenfalls in den 1970er Jahren (Schweden, Finnland, Norwegen) bzw. frühen 1980er Jahren (Dänemark) wurde die Elternzeit auf Väter ausgedehnt und ein Vaterurlaub (von 1 bis 2 Wochen) für die Zeit rund um die Geburt des Kindes eingeführt. Um die Inanspruchnahme der Elternzeit durch Väter zu erhöhen, wurde in den 1990er Jahren zusätzlich in allen

\footnotetext{
${ }^{18}$ In allen Ländern werden Einkommen ab einer bestimmten Höhe zu einem geringeren Prozentsatz kompensiert.

${ }^{19}$ In Finnland wurden gestaffelte Prozentsätze je nach Einkommenshöhe eingeführt.
} 
Ländern ein Teil der Elternzeit für den Vater reserviert (Finnland: 1 Woche; Dänemark: 2 Wochen; Norwegen: 4 Wochen; Schweden: 1 Monat). In Schweden wurde die Zeit weiter auf 2 Monate ausgedehnt; Finnland gewährt 12 Extra-Bonus Tage an Väter, die Elternzeit nehmen. In Dänemark wurde die Väterzeit 2002 abgeschafft. Die „Väterzeit“ kann ausschließlich vom Vater genutzt werden und ist nicht auf die Mutter übertragbar. In allen Ländern ist die Elternzeit/das Elterngeld auf einen - im Vergleich zu Deutschland - relativ kurzen Zeitraum von weniger als einem Jahr (Finnland), einem Jahr (Norwegen, Dänemark) und etwa 16 Monaten (Schweden; mit Reduktion des Elterngeldes für die letzten 3 Monate) beschränkt. Mütter und Väter haben ein individuelles Recht, Elternzeit mit Teilzeitarbeit zu kombinieren oder ihre Arbeitszeit nach der Elternzeit zu reduzieren. Dänemark führte $1994 \mathrm{im}$ Rahmen einer Umstrukturierung seiner aktiven Arbeitsmarktpolitik neben einer Kinderbetreuungszeit noch weitere bezahlte, berufliche Freistellungsmöglichkeiten wie Bildungsurlaub, Jobrotation und Berufsbildungsurlaub (sabbatical year) ein, die überwiegend von Frauen (häufig mit Kindern) in Anspruch genommen werden (Etherington 1998, S. 154; 157).

Trotz analoger Grundprinzipien, die die Sozial- und Familienpolitik in den nordischen Ländern bestimmen, weichen die fertilitätsbezogenen politischen Maßnahmen in den einzelnen Ländern voneinander ab. Finnland und Norwegen haben mit der Einführung einer bezahlten Kinderbetreuungszeit, die im Anschluss an die Elternzeit gewährt wird, die auf den Erhalt der Erwerbstätigkeit ausgerichtete Elternzeitpolitik aufgeweicht. Finnland begann als erstes der nordischen Länder 1985 eine bezahlte Kinderbetreuungszeit („home-care allowance") einzuführen, die 1990 voll zum Tragen kam (Ilmakunnas 1997; Hiilamo 2002; Simonen/Kovaleinen 1998). Nach der Elternzeit können finnische Eltern bis zum dritten Lebensjahr des Kindes ein Kinderbetreuungsgeld beziehen, sofern ihr Kind nicht in einer öffentlichen Kindertagesstätte betreut wird. Das Kinderbetreuungsgeld besteht aus einem einheitlichen Regelsatz, einem Zuschlag für jedes weitere Kind im Haushalt und einem vom Haushaltseinkommen abhängigen Zuschlag, der bezahlt wird, falls ein Elternteil selbst das Kind betreut. Die Kommunen können zusätzliche Beiträge zum Kinderbetreuungsgeld gewähren (Sipilä/Korpinen 1998, S. 266). Das Kinderbetreuungsgeld entsprach Anfang der 1990er Jahre in Summe etwa jenem Betrag, den eine Kommune für die öffentliche Betreuung des Kindes hätte aufwenden müssen (Sipilä/Korpinen 1998, S. 267). 1995 wurde es geringfügig, 1997 um 23\% gekürzt (Vikat 2004). Bis 1993 konnte das Kindergeld auch noch zusätzlich zu anderen Sozialleistungen, etwa dem Arbeitslosengeld, bezogen werden (Salmi/Lammi-Taskula 1999, S. 88f.). Norwegen führte 1998 eine ähnliche Regelung ein und gewährt ein Kinderbetreuungsgeld (für Kinder unter 3 Jahren), falls das Kind nicht ganztags in einer Kindertagesstätte betreut wird (Rønsen/Sundström 2002). Das Kinderbetreuungsgeld entsprach zur Zeit der Einführung dem staatlichen Zuschuss zu einer öffentlichen Betreuungseinrichtung, wurde in Folge jedoch reduziert (Rønsen 2004a, S. 279). ${ }^{20}$

\footnotetext{
${ }^{20}$ In Dänemark gab es von 1992 bis 2002 ebenfalls eine Kinderbetreuungszeit. Sie umfasste insgesamt 13 Wochen bis zum 8. Lebensjahr des Kindes, wobei unter 3-jährige Kinder keine Kindertagesstätte
} 
Während Finnland und Norwegen ihre Elternzeitsysteme in Richtung einer verlängerten familialen Betreuung des Kindes entwickelten, hat Schweden ein auf die Betreuungsbedürfnisse des Kindes und erwerbstätiger Eltern abgestimmtes flexibles Elternzeitsystem aufgebaut. ${ }^{21}$ Die schwedischen Regelungen erlauben es Eltern bereits seit den 1970er Jahren, ihre Elternzeit bis zum 8. Geburtstag des Kindes flexibel hinsichtlich des Zeitpunktes der Inanspruchnahme, der Dauer und der Teilung zwischen den Eltern zu gestalten. Die bezahlte Elternzeit kann auch tageweise oder auf Teilzeitbasis in Anspruch genommen werden. Eltern haben überdies das Recht, ihre Arbeitszeit bis zum Schuleintritt des Kindes um bis zu einem Viertel zu reduzieren. Die Gestaltung der Elternzeit, also die Aufteilung der Elternzeit bis zum 8. Geburtstag des Kindes, seine Nutzung als Vollzeit- oder Teilzeitelternzeit ebenso wie die Teilungen der Elternzeit zwischen den Eltern und die Möglichkeit der Reduktion der Arbeitszeit sind als individuelles Elternrecht verankert, sodass, anders als in Deutschland, auch bei einem Arbeitgeberwechsel das Anrecht auf die Nutzung der Elternzeit oder der Teilzeitmöglichkeit erhalten bleibt. Zudem gewährt Schweden seit 1980 einen Pflegeurlaub im Falle der Erkrankung des Kindes von bis zu 120 Tagen pro Kind und pro Jahr, wobei 60 Tage davon in Anspruch genommen werden können, falls die „übliche“ Betreuungsperson oder Betreuungsinstitution nicht in der Lage sind, das Kind zu betreuen (etwa durch Krankheit der Betreuungsperson oder weil die Betreuungsinstitution geschlossen ist). (Tabelle $1 \mathrm{im}$ Anhang gibt einen Überblick über die gegenwärtigen Regelungen zur Elternzeit in den nordischen Ländern und in Deutschland).

\section{(b) Institutionelle Kinderbetreuung in den nordischen Ländern}

Seit Ende der 1960er, Anfang der 1970er Jahre haben die nordischen Länder ihre institutionellen Kinderbetreuungsangebote von einem im Vergleich zu den kontintentaleuropäischen Ländern eher geringen Versorgungsgrad zu einem universellen Tagesbetreuungsangebot für Kinder aller Altersstufen ausgebaut. Der Ausbau war zunächst vom Bestreben getragen, die Frauenerwerbstätigkeit zu erhöhen. Doch bereits ab den 1970er Jahren rückte das Prinzip der sozialen und der Geschlechtergleichheit sowie der universellen Rechte aller in den Vordergrund (Sipilä et al. 1997, S. 33f., Waerness 1998; Simonen/Kovalainen 1998; Borchorst 2002; Leira 2002, S. 113ff.). Organisation und Finanzierung der institutionellen Kinderbetreuung in den nordischen Ländern garantieren ein nahezu flächendeckendes Angebot mit nur geringen Unterschieden in der Qualität (z.B.: im Verhältnis des qualifizierten Betreuungspersonals zur Anzahl der betreuten Kinder) und den

besuchen durften, ältere Kinder nur eine Teilzeitbetreuung in einer öffentlichen Kindertagesstätte (Rostgaard/Christoffersen/Weise 1999). Die Kinderbetreuungszeit wurde ab 2002 durch ein verlängertes und etwas flexibleres Elternzeitsystem abgelöst (NOSOSCO 2003, S. 10). Demnach dauert nun die Elternzeit 52 Wochen, inklusive der Zeit des Mutterschutzes sowie eines zweiwöchigen Vaterschaftsurlaubs aus Anlass der Geburt des Kindes. Ein Teil der verbleibenden 32 Wochen kann bis zum neunten Lebensjahr des Kindes in Anspruch genommen werden (MISSOC 2004).

${ }^{21} 1994$ wurde in Schweden ein Kinderbetreuungsgeld eingeführt, das jedoch nach den kurz darauf folgenden Wahlen und der Regierungsübernahme durch die Sozialdemokraten wieder abgeschafft wurde (Hiilamo 2002, S. 133). 
Kosten der Kinderbetreuung (Hank et al. 2004). In allen nordischen Ländern besteht ein Recht des Kindes auf einen institutionellen Betreuungsplatz, auch für Kinder unter 3 Jahren. Obwohl Finnland und Norwegen mit der Einführung eines Kinderbetreuungsgeldes für Eltern, deren Kind in den ersten Lebensjahren nicht oder nur zum Teil eine Kinderbetreuungsstätte besucht, einen finanziellen Anreiz geschaffen haben, Kinder zuhause zu betreuen, bleibt das Recht des Kindes auf einen institutionellen Betreuungsplatz erhalten.

Die nordischen Länder zählen - neben Frankreich - zu den Ländern mit dem höchsten Versorgungsgrad an institutioneller Betreuung in Europa. Von den 1- bis unter 3jährigen Kindern werden in den nordischen Ländern zwischen rund einem Drittel und drei Viertel aller Kinder institutionell betreut (2003: Dänemark: 78\%, Schweden: 65\%; Norwegen: 44\%; Finnland: 36\%). Wie die Statistik zeigt, ist in Finnland der Anteil der institutionell betreuten Kinder mit der Einführung des Kinderbetreuungsgeldes seit Anfang der 1990er Jahre gesunken (IIImakunas 1997; Anttonen/Sipilä 1996; Sipilä et al. 1997, Appendix). Dasselbe gilt - allerdings in geringerem Ausmaß - für Norwegen seit Einführung des Kinderbetreuungsgeldes im Jahre 1998 (Rønsen 2001). In Finnland stieg jedoch mit der Verbesserung der ökonomischen Lage in der zweiten Hälfte der 1990er Jahre die Zahl der unter 3-jährigen Kinder in Kinderbetreuungseinrichtungen wieder an (Anttonen 2001, S. 149f.). Auch in Norwegen ist der Anteil der institutionell betreuten unter 3-jährigen Kinder wieder im Ansteigen (NOSOSCO 2005). Von den Kindern im Kindergartenalter (3 bis Schuleintritt) besuchen in Finnland 68\%, in Norwegen $85 \%$ und in Dänemark und Schweden 94\% eine Kinderbetreuungseinrichtung. Außerschulische Betreuung wird von schulpflichtigen Kindern insbesondere in Dänemark (66\%) und Schweden (59\%) in Anspruch genommen (NOSOSCO 2005).

(c) Exkurs: Tagesbetreuungsausbaugesetz und die Elternzeit- und Elterngeldregelungen in Deutschland ab 2007

Die niedrigen Geburtenraten in Deutschland, die hohe Kinderlosigkeit unter hoch qualifizierten Frauen, die EU-Vorgaben hinsichtlich des Elternurlaubs (Richtlinie zum Elternurlaub 1996, 96/34/EWG), der Anhebung der Frauenerwerbstätigkeit auf 60\% (bis 2010) und des Ausbaus an Kinderbetreuungseinrichtungen, um bis 2010 eine Betreuungsquote von 30\% für unter 3-jährige Kinder und 90\% für 3- bis 6-jährige Kinder zu erreichen (Lissabonn-Strategie), bildeten in Deutschland einen Ansatzpunkt, Veränderungen in der Tagesbetreuungspolitik und der Elternzeitpolitik durchzuführen. ${ }^{22}$ Die Kinderbetreuungsangebote, insbesondere für unter 3-jährige Kinder, sollen bedarfsgerecht ausgebaut werden. Bis 2010 sollen mindestens 230.000 zusätzliche Plätze für Kinder dieser Altersgruppe geschaffen werden, um es Eltern mit Kleinkindern zu ermöglichen, eine Erwerbstätigkeit auszuüben oder an einer Bildungsmaßnahme teilzunehmen (Tagesbetreuungsausbaugesetz 2004; Leitner 2005). Seit 2006 können Erwerbstätige

\footnotetext{
${ }^{22}$ Zur EU als Anstoß zu familienpolitischen Veränderungen, siehe Falkner et al. (2005); zum innerdeutschen Reformprozess siehe Bothfeld (2005).
} 
Kinderbetreuungskosten für Kinder bis zum Alter von 14 Jahren, Nicht-erwerbstätige Kinderbetreuungskosten für Kinder zwischen 3 und 6 Jahren steuerlich bis zu einer bestimmten Gesamtsumme geltend machen (Gesetz zur steuerlichen Förderung von Wachstum und Beschäftigung 2006).

Während die Diskussion über die nicht-familiäre Kinderbetreuung vorwiegend im arbeitsmarktpolitischen Kontext geführt wurde (Leitner 2005), stehen geburtenbezogene Aspekte bei der Reform der Elternzeitregelungen im Vordergrund. Nach den Vereinbarungen der Koalitionsparteien (Stand: Juni 2006) soll für Geburten ab 2007 das Erziehungsgeld durch ein einkommensabhängiges Elterngeld ersetzt werden. Die Höhe des Elterngeldes soll 67\% des vorangegangen Einkommens, max. $€ 1.800$ pro Monat, betragen. Die Bezugsdauer soll 12 Monate betragen, falls nur ein Elternteil das Elterngeld in Anspruch nimmt, und 14 Monate, falls auch der andere Elternteil Elternzeit in Anspruch nimmt („Vätermonate“). Eltern, die vor der Geburt ihres Kindes nicht erwerbstätig waren, erhalten für die Dauer von 12 Monaten einen Sockelbetrag, der dem bisherigen Erziehungsgeld ( $€ 300$ pro Monat) entspricht. ${ }^{23}$

Elterngeldbeziehende, die ein zweites oder weiteres Kind innerhalb von 24 Monaten nach ihrem vorangegangenen haben, und zwischen den Geburten nicht oder nur teilzeiterwerbstätig sind, sollen einen "Geschwisterbonus" erhalten. Dieser soll 50\% der Differenz zwischen dem vorangegangenen und dem aktuellen Elterngeld betragen (Bundesregierung 2006).

Mit den beabsichtigten Änderungen der Elternzeit und des Erziehungsgeldes wird eine Abkehr von der bisherigen Elternzeitpolitik in Deutschland und eine Hinwendung zu den Elternzeitmodellen der nordischen Länder vollzogen. Die Angleichung des deutschen Erziehungsgeldes an die Elterngeldmodelle der nordischen Länder ist nicht zuletzt von dem Bestreben getragen, die Bereitschaft von Frauen und Männern, ein Kind zu haben, zu unterstützen. Die im europäischen Vergleich relativ hohen Gesamtfertilitätsraten der nordischen Länder nähren in Öffentlichkeit und Politik die Hoffnung, durch eine den nordischen Ländern ähnliche Elternzeitpolitik der geringen Geburtenneigung in Deutschland entgegenzuwirken. Umso bedeutsamer ist die Frage, welche Wirkungen die Elternpolitik in den nordischen Ländern auf das Geburtenverhalten ausübte.

\subsection{Wirkungen von Familienpolitik auf Fertilität}

Die hohen Fertilitätsraten in den nordischen Ländern werden zwar häufig mit der Wohlfahrtsstaat- und der Familienpolitik dieser Länder in Zusammenhang gebracht. Doch Vergleiche der Fertilitätsraten, der Raten der Frauenerwerbstätigkeit und der sozial- bzw.

\footnotetext{
${ }^{23}$ Weitere geplante Aspekte der neuen Elterngeldregelungen sind: Bei geringem Einkommen (unter $€$ 1000 pro Monat) soll sich das Elterngeld auf bis zu 100\% des vorangegangenen Einkommens erhöhen, wobei bei der Berechnung auch das Einkommen des Partners berücksichtigt werden soll. Das Elterngeld kann bei gleicher Bezugssumme auf zwei Jahre erstreckt werden. Die Möglichkeit einer gesetzlich geschützten Erwerbsunterbrechung (Elternzeit) bis zu drei Jahren soll erhalten bleiben.
} 
familienpolitischen Ausgestaltung der Wohlfahrtsstaaten zwischen den nordischen Ländern, den angelsächsischen Ländern und den kontinentaleuropäischen Ländern lassen einen solchen Zusammenhang nicht zwingend erscheinen. So weisen auch die angelsächsischen Länder ebenso wie Frankreich, Belgien und in jüngerer Zeit auch die Niederlande relativ hohe Fertilitätsraten auf, obwohl ihre wohlfahrtsstaatliche Politik sich deutlich von jener der nordischen Länder unterscheidet. Studien, die die Fertilitätsentwicklung auf der Basis von Gesamtfertilitätsraten miteinander vergleichen, kommen ebenfalls zu keinem eindeutigen Ergebnis in Bezug auf die Wirkungen von Familienpolitik (Neyer 2003, Appendix), lassen jedoch einen Zusammenhang zwischen dem Grad der Versorgung mit Kinderbetreuungseinrichtungen und der Fertilitätsrate erkennen (Castles 2003). Ein Grund für die mangelnden Belege einer Wirkung von Familienpolitik auf Fertilität liegt, wie bereits in der Einleitung erwähnt, darin, dass die Gesamtfertilitätsraten das Geburtenverhalten nur unzureichend wiederspiegeln und damit auch die Wirkungen von Sozial- und Familienpolitik auf dieses Verhalten nicht eruiert werden können. Für eine Darstellung der Wirkungen von familienpolitischen Maßnahmen auf die Fertilität bieten Individualdaten, wie die Registerdaten der nordischen Länder, eine bessere Studienbasis. Wie Analysen zu den nordischen Ländern zeigen, haben familienpolitische Maßnahmen in diesen Ländern tatsächlich einen Einfluss auf das Geburtenverhalten ausgeübt. Doch können die Wirkungen in unterschiedlichen Kontexten höchst unterschiedlich sein.

In allen nordischen Ländern, ausgenommen Schweden, hat die Einführung eines einkommensbezogenen Elterngeldes mit annähernden Einkommensersatzquoten die Neigung ein zweites Kind zu haben, verstärkt (siehe Grafik 3). In Norwegen, wo es das Elterngeld (mit 100\% Einkommensersatz) seit 1978 gibt, stoppte der Rückgang der Zweigeburtenrate. In Finnland stieg mit der Erhöhung des Elterngeldes auf einen annähernden Lohnersatz 1982 die Zweitgeburtenrate an. Auch in Dänemark, das seine Mutterschafts- und Elternzeitregelungen stufenweise zwischen 1981 und 1985 verbesserte, kam es zu einer Trendumkehr bei den Zweitgeburtenraten. Lediglich in Schweden lässt sich keine unmittelbare Veränderung im Geburtenverhalten von Müttern mit einem Kind erkennen. Dort setzte der Anstieg der Zweitgeburtenraten etwas verzögert ein. Ähnliche Entwicklungen wie bei den Zweitgeburten gibt es auch bei den Drittgeburtenraten und (mit Ausnahme Schwedens und Norwegens) bei den Erstgeburtenraten von über 30-jährigen Frauen. Der nach Einführung des Elterngeldes in fast allen nordischen Ländern zu beobachtende länger dauernde Anstieg der Geburtenraten kann jedoch nicht allein dieser Maßnahme zugeschrieben werden. Mehrere andere Faktoren, wie die Arbeitsmarktlage, die aktive Förderung der Frauenerwerbstätigkeit, die Gleichheitspolitik und die Versorgung mit Kinderbetreuungseinrichtungen sind dabei von entscheidender Bedeutung (Rønsen 2004a; 2004b). Doch scheint die Einführung des Elterngeldes in den nordischen Ländern eine Veränderung im Geburtenverhalten in Gang gesetzt oder eine bereits begonnene Veränderung verstärkt zu haben. 
Eine messbare Wirkung auf das Geburtenverhalten hatte die Einführung der "Geschwindigkeitsprämie“ in Schweden in den 1980er Jahren. Hoem (1993), Andersson (1999) und Andersson/Hoem/Duvander (2006) haben gezeigt, dass die Änderung der Elterngeldregelung, die Eltern, die ihr zweites oder weiteres Kind innerhalb eines bestimmten Zeitraumes nach dem vorangegangen Kind bekommen, finanziell bevorzugt, zu einer Verkürzung des Geburtenabstandes geführt hat. Dies bewirkte einen deutlichen Anstieg der Zweitgeburtenraten und Drittgeburtenraten, insbesondere nach Ausdehnung des zulässigen Zeitraumes zwischen zwei Geburten von 24 auf 30 Monate im Jahre 1986 (siehe auch Grafik 3 und Grafik 4). Der Rückgang der Fertilität in den 1990er Jahren war nicht mit einer grundlegenden Änderung des Verhaltens in Bezug auf den Geburtenabstand verbunden. Noch heute bekommen Frauen in Schweden ihr zweites oder weiteres Kind deutlich früher nach dem vorangegangenen Kind als vor der Einführung der Maßnahme.

Der Rückgang der Gesamtfertilitätsrate in Schweden in der ersten Hälfte der 1990er Jahre ist vor allem auf den Anstieg der Arbeitslosigkeit während der ökonomischen Krise zu Beginn der 1990er Jahre und die Zunahme an nicht-erwerbstätigen oder sich in Bildungsmaßnahmen befindenden Frauen zurückzuführen. Nicht-erwerbstätige oder sich in Ausbildung befindende Frauen werden generell seltener Mütter als erwerbstätige Frauen. Der Anstieg der Nicht-erwerbstätigkeit unter Frauen bewirkte somit einen Rückgang der Fertilitätsraten; mit der Reduktion der Arbeitslosigkeit und dem Anstieg der Erwerbstätigkeit von Frauen in der zweiten Hälfte der 1990er Jahre stiegen auch die Fertilitätsraten wieder an. Dieses „pro-zyklische“ Geburtenverhalten (Andersson 2000; 2002) schwedischer Frauen wird vor allem auf die starke Erwerbsbezogenheit der Elternzeitregelungen und des Elterngeldes in Schweden zurückgeführt (Andersson 2002). Die Reduktion des Elterngeldes (von 90\% auf 75\%) und anderer Sozialleistungen (wie die Reduktion des Arbeitslosengeldes) in der Zeit der ökonomischen Krise haben die Krisenwirkungen auf die Fertilitätsentscheidungen noch verstärkt, sodass trotz der fertilitätsbegünstigenden Elterngeldregelungen für Eltern, die ein zweites oder weiteres Kind haben wollten, ein Rückgang der zweiten und weiteren Geburtenraten zu verzeichnen war (ebenda).

Eine gänzlich andere Wirkung hatte das Kinderbetreuungsgeld in Finnland, dessen stufenweise Einführung gerade zur Zeit der ökonomischen Krise Anfang der 1990er Jahre beendet war. Vikat (2004) zeigte, dass die Inanspruchnahme des Kinderbetreuungsgeldes die Neigung zu einem zweiten Kind nicht erhöhte und nur zu einem geringen Teil einen Einfluss auf die Drittgeburtenraten hatte. Die Arbeitsmarktsituation der Frauen hatte einen deutlich größeren Einfluss auf ihre Bereitschaft, ein (weiteres) Kind zu bekommen als die Möglichkeit, ein Kinderbetreuungsgeld zu beziehen. Das Kinderbetreuungsgeld ermöglichte es Frauen, die arbeitslos wurden, die Zeit der ökonomischen Krise und der reduzierten Chancen, einen Arbeitsplatz zu finden, zu überbrücken (ebenda). Allerdings verringerte die Inanspruchnahme eines Kinderbetreuungsgeldes in Finnland die Wiedereinstiegschancen von Frauen in den Arbeitsmarkt, insbesondere den Wiedereinstieg auf Vollzeitbasis, der vorherrschenden Arbeitszeitform für Frauen und Männer in Finnland (Rønsen/Sundström 
2002). Eine ähnlich negative Wirkung des Kinderbetreuungsgeldes auf den Wiedereinstieg in den Arbeitsmarkt wurde auch für Norwegen festgestellt (ebenda).

Keine oder nur geringe Wirkungen fanden Forscherlnnen hinsichtlich des Zusammenhangs zwischen der Versorgung mit Kinderbetreuungseinrichtungen und der Neigung von Frauen, ein weiteres Kind zu haben (Kravdal 1996; Andersson/Duvander/Hank 2005). Diese geringe Wirkung ist jedoch auf den relativ hohen Versorgungsgrad mit Kinderbetreuungseinrichtungen und die geringen Unterschiede im Angebot, der Qualität und den Kosten institutioneller Betreuung in den nordischen Ländern zurückzuführen (ebenda).

Dass gleichheitsbezogene Maßnahmen jedoch eine positive Wirkung auf das Geburtenverhalten ausüben können, belegen Studien zur Inanspruchnahme von Vaterschaftsurlaub und Studien über die Effekte von Einkommen bei Paaren. Schwedische Familien, in denen der Vater (auch nur einen geringen) Teil der Elternzeit beim ersten Kind in Anspruch nahm, hatten eher ein zweites Kind als solche, in denen der Vater keine Elternzeit in Anspruch genommen hatte (Oláh 2003; Duvander/Andersson 2006). Desgleichen zeigen schwedische Studien, dass das Einkommen der Frau eher einen Einfluss auf die Bereitschaft zu einem (weiteren) Kind ausübt als das Einkommen des Mannes. Je höher das Einkommen der Frau, umso eher tendiert sie dazu, ein (weiteres) Kind zu haben (Andersson/Duvander/Hank 2004). Zwar steigt auch mit dem Einkommen des Mannes die Chance zur (weiteren) Vaterschaft; doch in der Entscheidung für ein drittes Kind ist in allen Einkommensgruppen das Einkommen der Frau von größerer Bedeutung als das Einkommen des Mannes (ebenda). Dieses Ergebnis ist vor allem auch vor dem Hintergrund relevant, dass mit einer Verlängerung der Elternzeit die Dauer der Berufsunterbrechung für Frauen nach einer Geburt steigt (Rønsen/Sundström 2002 für Norwegen, Schweden und Finnland). Wesentlich scheint daher für die Entscheidung zu einem (weiteren) Kind auch zu sein, in welchem Ausmaß Frauen sowohl vor als auch nach einer Geburt die Möglichkeit geboten wird, ein adäquates Erwerbseinkommen zu erzielen (siehe auch Kravdal 1994; Rønsen 2004a; 2004b). Die Entprivatisierung von Betreuung durch ein umfassendes Angebot an Kindertagesstätten und eine auf Erwerbstätigkeit aller ausgerichtete Arbeitsmarktpolitik scheinen dafür eine Voraussetzung zu sein, ebenso wie eine Politik, die Geschlechterungleichheiten am Arbeitsmarkt und in der privaten Betreuungsarbeit reduziert.

Grundsätzlich, so lässt sich aus diesen Ergebnissen schließen, können die Wirkungen von familienpolitischen Maßnahmen auf die Fertilität nur im Kontext der ökonomischen Entwicklung eines Landes, der Arbeitsmarktwirkungen und der Geschlechterwirkungen der Familienpolitik beurteilt werden. Doch scheinen die nordischen Länder mit ihrer Wohlfahrtsstaat-, Arbeitsmarkt- und Familienpolitik, die die Gleichheit sowohl hinsichtlich der Erwerbstätigkeit, der Einkommen als auch der Betreuung fördert, einen politischen Weg eingeschlagen zu haben, der es Frauen und Männern aller sozialen Gruppen ermöglicht, in höherem Maße ein Kind oder ein weiteres Kind zu haben als in Deutschland. 


\section{Literatur:}

Adserà, Alícia (2004): Changing fertility rates in developed countries. The impact of labor market institutions. In: Journal of Population Economics 17, S. 17-43.

Ahn, Namkee/Mira, Pedro (2002): A note on the changing employment relationship between fertility and female employment rates in developed countries. In: Journal of Population Economics 15, 4, S. 667-682

Andersson, Gunnar (1999): Childbearing trends in Sweden 1961-1997. In: European Journal of Population 15, S. 1-24.

Andersson, Gunnar (2000): The impact of labor-force participation on childbearing behavior. Pro-cyclical fertility in Sweden during the 1980s and the 1990s. In: European Journal of Population 16, S. 293-333.

Andersson, Gunnar (2002): Fertility development in Norway and Sweden since the early 1960s. In: Demographic Research 6, 4, S. 67-86: http://www.demographicresearch.org/volumes/vol6/4/6-4.pdf

Andersson, Gunnar (2004): Childbearing developments in Denmark, Norway and Sweden from the 1970s to the 1990s. A comparison. In: Demographic Research S3, 7, S. 155-176: http://www.demographic-research.org/special/3/7/S3-7.pdf

Andersson, Gunnar/Duvander, Ann-Zofie/Hank, Karsten (2004): Do child care characteristics influence continued childbearing in Sweden? An investigation of the quantity, quality, and price dimension. In: Journal of European Social Policy 14, 4, S. 407-418.

Andersson, Gunnar/Duvander, Ann-Zofie/Hank, Karsten (2005): Erwerbsstatus und Familienentwicklung in Schweden aus paarbezogener Perspektive. In: Tölke, Angelika/Hank, Karsten (Hrsg.): Männer - das „vernachlässigte“ Geschlecht in der Familienforschung. Sonderheft 4 der Zeitschrift für Familienforschung. Wiesbaden: VS Verlag für Sozialwissenschaften, S. 220-234.

Andersson, Gunnar/Hoem, Jan M./Duvander, Ann-Zofie (2006): Social differentials in speedpremium effects in childbearing in Sweden. In: Demographic Research 14, 4, S. 51-70: http://www.demographic-research.org/volumes/vol14/4/14-4.pdf

Anttonen, Annelie (2001): The politics of social care in Finland. Child and elder care in transition. In: Daly, Mary (Hrsg.): Care work. The quest for security. Geneva: ILO, S. 143-158.

Anttonen, Annelie/Sipilä, Jorma (1996): European social care services. Is it possible to identify models? In: Journal of European Social Policy 6, 2, S. 87-100. 
Bergqvist, Christina (1999): Childcare and parental leave models. In: Bergqvist, Christina/Borchorst, Anette/Christensen, Ann-Dorte/Raaum, Nina C./Ramstedt-Silén, Viveca/Styrkársdóttir, Auður (Hrsg.): Equal democracies? Gender and politics in the Nordic countries. Oslo: Scandinavian University Press, S. 121-136.

Bergqvist, Christina/Borchorst, Anette/Christensen, Ann-Dorte/Raaum, Nina C./RamstedtSilén, Viveca/Styrkársdóttir, Auður (Hrsg.) (1999): Equal democracies? Gender and politics in the Nordic countries. Oslo: Scandinavian University Press.

Blossfeld, Hans-Peter/Huinink, Johannes (1991): Human capital investment or norms of role transition? How women's schooling and career affect the process of family formation. In: American Journal of Sociology 97, 1, S. 143-168.

Borchorst, Anette (2002): Danish child care policy. Continuity rather than radical change. In: Michel, Sonya/Mahon, Rianne (Hrsg.): Child care policy at the crossroads. Gender and welfare state Restructuring. London: Routledge, S. 267-286.

Bothfeld, Silke (2005): Vom Erziehungsurlaub zur Elternzeit: Politisches Lernen im Reformprozess. Frankfurt a. M.: Campus Verlag.

Brewster, Karin L./Rindfuss, Ronald R. (2000): Fertility and women's employment in industrialized nations. In: Annual Review of Sociology 26, S. 271-296.

Bundesministerium für Familie, Senioren, Frauen und Jugend (2002): Erziehungsgeld, Elternzeit. Das neue Bundeserziehungsgeld für Eltern mit Kindern ab dem Geburtsjahrgang 2001. Berlin: BMFSFJ.

Bundesregierung (2006): Das Elterngeld kommt! http://www.bundesregierung.de/413.1016628/artikel/Das-Elterngeld-kommt.htm

Castles, Francis G. (2003): The world turned upside down: Below replacement fertility, changing preferences and family-friendly public policy in 21 OECD Countries. In: Journal of European Social Policy 13, 3, S. 209-227.

Council of Europe (2004): Demographic Yearbook 2004. CD Supplement. Strassburg: Council of Europe.

Deutsches Jugendinstitut (2002): Zahlenspiegel. Daten zu Tageseinrichtungen für Kinder. München: Deutsches Jugendinstitut: http://cgi.dji.de/bibs/zahlenspiegel gesamt.pdf

Deven, Fred/Moos, Peter (Hrsg.) (2005): Leave policies and research: Reviews and country notes. CBGS-Werkdocument 2005/3. Brussels. 
Duvander, Ann-Zofie/Andersson, Gunnar (2006): Gender equality and fertility in Sweden. A study on the impact of the father's uptake of parental leave on continued childbearing. In: Marriage and Family Review 39 (im Erscheinen).

Engelhardt, Henriette/Kögel, Tomas/Prskawetz, Alexia (2004): Fertility and women's employment reconsidered. A macro-level time-series analysis for developed countries, 19602000. In: Population Studies 58, 1, S. 109-120.

Esping-Andersen, Gøsta (1990): The three worlds of welfare capitalism. Cambridge: Princeton University Press.

Esping-Andersen, Gøsta (1999): Social foundations of postindustrial economies. Oxford: Oxford University Press.

Esping-Andersen, Gøsta (2002): A new gender contract. In: Esping-Andersen, Gøsta/Gallie, Duncan/Hemerijck, Anton/Myles, John (Hrsg.): Why we need a new welfare state. Oxford: Oxford University Press, S. 68-95.

Etherington, David (1998): From welfare to work in Denmark: an alternative to free market policies? In: Policy \& Politics 26, 2, S. 147-161.

Falkner, Gerda/Treib, Oliver/Hartlapp, Miriam/Leibner, Simone (2005): Complying with Europe: EU harmonisation and soft law in the member states. Cambridge: Cambridge University Press.

Gesetz zur steuerlichen Förderung von Wachstum und Beschäftigung vom 26.04.2006, BGBI. I, Nr. 22, vom 05.05.2006, S. $1091 \mathrm{ff}$.

Gornick, Janet C. (1999): Gender equality in the labour market. In: Sainsbury, Diane (Hrsg.): Gender and welfare state regimes. Oxford: Oxfore University Press, S. 210-242.

Grünheid, Evelyn (2004): Junge Frauen in Deutschland. Bei hoher Ausbildung kinderlos? In: Psychosozial 95, 1, S. 35-46.

Hank, Karsten/Andersson, Gunnar/Duvander, Ann-Zofie/Kreyenfeld, Michaela/Spieß, Katharina (2004): Öffentliche Kinderbetreuung und individuelle Fertilitätsentscheidungen in Deutschland und Schweden. In: Stöbel-Richter, Yve/Brähler, Elmar (Hrsg.): Demographischer und sozialer Wandel. Gießen: Psychosozialverlag, S. 47-57.

Hank, Karsten/Kreyenfeld, Michaela/Spieß Katharina (2004): Kinderbetreuung und Fertilität in Deutschland. Zeitschrift für Soziologie 33, 3, S. 228-244. 
Hiialmo, Heikki (2002): The rise and fall of Nordic family policy? Historical development and changes during the 1990s in Sweden and Finland. STAKES (National Research and Development Centre for Welfare and Health) Research Report 125, Helsinki: STAKES.

Hoem, Britta (1993): The compatibility of employment and childbearing in contemporary Sweden. In: Acta Sociologica 36, S. 101-120.

Hoem, Britta/Hoem, Jan M. (1989): The impact of women's employment on second and third births in modern Sweden. In: Population Studies 43, 1, S. 47-67.

Hoem, Jan M. (1990): Social policy and recent fertility change in Sweden. In: Population and Development Review 16, S. 735-748.

Hoem, Jan M. (1993): Public Policy as the Fuel of Fertility. Effects of a Policy Reform on the Pace of Childbearing in Sweden in the 1980s. In: Acta Sociologica 36, S. 19-31.

Hoem, Jan M./Neyer, Gerda/Andersson, Gunnar (2006a): Education and childlessness. The relationship between educational field, educational level, and childlessness among Swedish women born in 1955-59. In: Demographic Research 14, 15, S. 331-380: http://www.demographic-research.org/Volumes/Vol14/15/14-15.pdf

Hoem, Jan M./Neyer, Gerda/Andersson, Gunnar (2006b): Educational attainment and ultimate fertility among Swedish women born 1955-59 In: Demographic Research 14, 16, S. 381-404: http://www.demographic-research.org/volumes/vol14/16/14-16.pdf

Hoem, Jan M./Prskawetz, Alexia/Neyer, Gerda (2001): Autonomy or conservative adjustment? The effect of public policies and educational attainment on third births in Austria, 1975-96. In: Population Studies 55, S. 249-261. Als längere Version unter: http://www.demogr.mpg.de/Papers/Working/wp-2001-016.pdf

Huinink, Johannes (2002): Polarisierung der Familienentwicklung in europäischen Ländern im Vergleich. In: Schneider, Norbert F./Matthias-Beck, Heike (Hrsg.): Elternschaft heute. Gesellschaftliche Rahmenbedingungen und individuelle Gestaltungsaufgaben. Zeitschrift für Familienforschung, Sonderheft 2, Opladen, S. 49-73.

IImakunnas, Seija (1997): Public policies and childcare choice. In: Persson, Inga/Jonung, Christina (Hrsg.): Economics of the family and family policies. London: Routledge, S. 179193.

Knudsen, Lisbeth B. (2002): An overview of recent fertility trends and family policies in Denmark. In: Carling Jørgen (Hrsg.): Nordic demography. Trends and differentials. Scandinavian Population Studies, Vol. 13. Oslo: Unipub forlag and the Nordic Demographic Society, S. 131-156. 
Kolbe, Wiebke (2002): Elternschaft im Wohlfahrtsstaat. Schweden und die Bundesrepublik im Vergleich 1945-2000. Frankfurt a. M.: Campus Verlag.

Kravdal, Øystein (1992): The emergence of a positive relation between education and third birth rates in Norway with supportive evidence from the United States. In: Population Studies 46, 3, S. 459-475.

Kravdal, Øystein (1994): The importance of economic activity, economic potential and economic resources for the timing of first birth in Norway. In: Population Studies 48, 2, S. 249267.

Kravdal, Øystein (1996): How the local supply of day-care centers influences fertility in Norway. A parity-specific approach. In: Population Research and Policy Review 15, S. 201 218.

Kravdal, Øystein (2001): The high fertility of college educated women in Norway: An artifact of the separate modelling of each partiy transition. In: Demographic Research 5, 6, S. 185-216: http://www.demographic-research.org/volumes/vol5/6/5-6.pdf

Kreyenfeld, Michaela (2002): Time-squeeze, partner effect or self-selection? An investigation into the positive effect of women's education on second birth risks in West Germany. In: Demographic Research 7, 2, S. 15-47: http://www.demographicresearch.org/Volumes/Vol7/2/7-2.pdf

Kreyenfeld, Michaela (2004a): Politikdiskussion fehlt verlässliche statistische Grundlage. Datenprobleme in der Demografie am Beispiel der Kinderlosigkeit in Deutschland. In: Demografische Forschung aus erster Hand 1, 3, S. 4: http://www.demografischeforschung.org/archiv/defo0403.pdf

Kreyenfeld, Michaela (2004b): Fertility decisions in the FRG and GDR. An analysis with data from the German fertility and family survey. In: Demographic Research S3, 11, S. 275-318: http://www.demographic-research.org/special/3/11/S3-11.pdf

Langan, Mary/Ostner, Ilona (1991): Geschlechterpolitik im Wohlfahrtsstaat. Aspekte im internationalen Vergleich. In: Kritische Justiz, S. 302-317.

Lappegård, Trude (2002): Educational attainment and fertility pattern among Norwegian women. Statistics Norway. Documents 2002/18.

Lappegård, Trude/Rønsen, Marit (2005): The multifaceted impact of education on entry into motherhood. In: European Journal of Population 21, S. 31-49.

Leira, Arnlaug (2002): Working parents and the welfare state. Family change and policy reform in Scandinavia. Cambridge: Cambridge University Press. 
Leitner, Sigrid (2005): Kind und Karriere für alle? Geschlechts- und schichtspezifische Effekte rot-grüner Familienpolitik. In: Blätter für deutsche und internationale Politik 8, S. 958-964.

Lewis, Jane (1992): Gender and the development of welfare regimes. In: Journal of European Social Policy 3, S. 159-173.

Meyers, Marcia K./Gornick, Janet C./Ross, Katherin E. (1999): Public childcare, parental leave, and employment. In: Sainsbury, Diane (Hrsg.): Gender and welfare state regimes. Oxford: Oxford University Press, S. 117-146.

Missoc (2001 und diverse weitere Jahre): Social protection in the member states in the EU member states and the European economic area: http://europa.eu.int/comm/employment social/missoc/index en.html

New Cronos (2001). Eurostat Datenbasis (CD-Rom).

Neyer, Gerda (2003): Family policies and low fertility in Western Europe. In: Journal of Population and Social Security (Population) 1, Supplement, S. 46-93. (Verfügbar auch als MPIDR Working Paper WP-2003-021: http://www.demogr.mpg.de/papers/working/wp-2003021.pdf)

Neyer, Gerda/Hoem, Jan M. (2006): Educational fields and childlessness in Austria. MPIDR Working Paper (im Erscheinen).

NOSOSCO (2002 und weitere Jahre): Social protection in the Nordic countries 2000. Scope, expenditure and financing. Nordic Social-Statistical Committee no 18:02. Copenhagen: http://www.nom-nos.dk/nososco.htm

OECD (1998): Labour force statistics 1977-1997. Paris: OECD.

OECD (2004): Labour force statistics indicators. Paris: OECD.

OECD (2001): OECD Employment outlook. Chapter 4: Balancing work and family life: Helping parents into paid employment. Paris: OECD.

Oláh, Livia Sz. (2003): Gendering fertility: Second births in Sweden and Hungary. In: Population Research and Policy Review 22, S. 171-200.

Rønsen, Marit (1999): Impacts on fertility and female employment of parental leave programs. Evidence from three Nordic countries. Beitrag für die European Population Conference, Den Haag, Niederlande, 30. August - 3. September 1999. 
Rønsen, Marit (2001): Market work, childcare and the division of household labour. Adaptations of Norwegian mothers before and after the cash-for-care reform. Reports 2001/13, Statistics Norway.

Rønsen, Marit (2004a): Fertility and family policy in Norway - A reflection on trends and possible connections. In: Demographic Research 10, 10, S. 265-286: http://www.demographic-research.org/Volumes/Vol10/10/10-10.pdf

Rønsen, Marit (2004b): Fertility and public policies - Evidence from Norway and Finland. In: Demographic Research 10, 6, S. 143-170: http://www.demographicresearch.org/Volumes/Vol10/6/10-6.pdf

Rønsen, Marit/ Sundström, Marianne (2002): Family policy and after-birth employment among new mothers - a comparison of Finland, Norway and Sweden. In: European Journal of Population 18, S. 121-152.

Rostgaard, Tine/Christoffersen, Morgens N./Weise, Hanne (1999): Parental leave in Denmark. In: Moss, Peter/Deven, Fred (Hrsg.): Parental leave: Progress or pitfall? Research and policy issues in Europe. NIDI/ CBGS Publications 35, Brussels: Vlaamse Gemeenschap,

Sainsbury, Diane (1999a): Gender, policy regimes, and politics. In: Sainsbury, Diane (Hrsg.): Gender and welfare state regimes. Oxford: Oxford University Press, S. 245-275.

Sainsbury, Diane (1999b): Taxation, family responsibilities, and employment. In: Sainsbury, Diane (Hrsg.): Gender and welfare state regimes. Oxford: Oxford University Press, S. 185209.

Salmi, Minna/Lammi-Taskula, Johanna (1999): Parental leave in Finland. In: Moss, Peter/Deven, Fred (Hrsg.) (1999): Parental leave: Progress or pitfall? Research and policy issues in Europe. NIDI/ CBGS Publications 35. Brussels: Vlaamse Gemeenschap, S. 85-121.

Simonen, Leila/Kovalainen, Anne (1998): Paradoxes of social care restructuring. The Finnish case. In: Lewis, Jane (Hrsg.): Gender, social care and welfare state restructuring in Europe. Aldershot: Ashgate, S. 229-255.

Sipilä, Jorma/Andersson, Margit/Hammarqvist, Sten-Erik/Nordlander, Lars/Rauhala, PirkkoLiisa/Thomsen, Kåre/Nielsen, Hanne Warming (1997): A multitude of universal public services - how and why four Scandinavian countries get their social care service model? In: Sipilä, Jorma (Hrsg.): Social care services. The key to the Scandinavian welfare model. Aldershot: Ashgate, S. 27-50.

Sipilä, Jorma/Korpinen, Johanna (1998): Cash versus child care services in Finland. In: Social Policy \& Administration 32, 2, S. 263-277. 
Skrede, Kari (2003): Opening the black box. Some preliminary findings on early transition to parenthood and the role of cohabiting unions in early family formation in Norway. Beitrag für den Workshop: Das vernachlässigte Geschlecht in der Familienforschung: Analysen zum Heirats- und Geburtenverhalten von Männern in Deutschland. Max-Planck-Institut für demografische Forschung, 27./28. Februar, Rostock.

Tagesbetreuungsausbaugesetz (2004): Gesetz zum qualitätsorientierten und bedarfsgerechten Ausbau der Tagesbetreuung für Kinder (Tagesbetreuungsausbaugesetz TAG) vom 27.12.2004, BGBI. I, Nr. 75, vom 27.12.2005, S. 3852ff.

Trappe, Heike (1995): Emanzipation oder Zwang? Frauen in der DDR zwischen Beruf, Familie und Sozialpolitik. Berlin: Akademie-Verlag.

Vikat, Andres (2004): Women's labor force attachment and childbearing in Finland. In: Demographic Research S3, 8, S. 177-212: http://www.demographicresearch.org/special/3/8/S3-8.pdf

Waerness, Kari (1998): The changing 'welfare mix' in childcare and care for the frail elderly in Norway. In: Lewis, Jane (Hrsg.): Gender, social care and welfare state restructuring in Europe. Aldershot: Ashgate, S. 207-228.

Wirth, Heike/Dümmler, Kerstin (2004): Zunehmende Tendenz zu späteren Geburten und Kinderlosigkeit bei Akademikerinnen. Eine Kohortenanalyse auf der Basis von Mikrozensusdaten. In: Informationsdienst Soziale Indikatoren (ISI) 32, S. 1-6. 
Grafik 1: Standardisierte Erstgeburtenraten kinderloser unter 30-jähriger Frauen in den nordischen Ländern

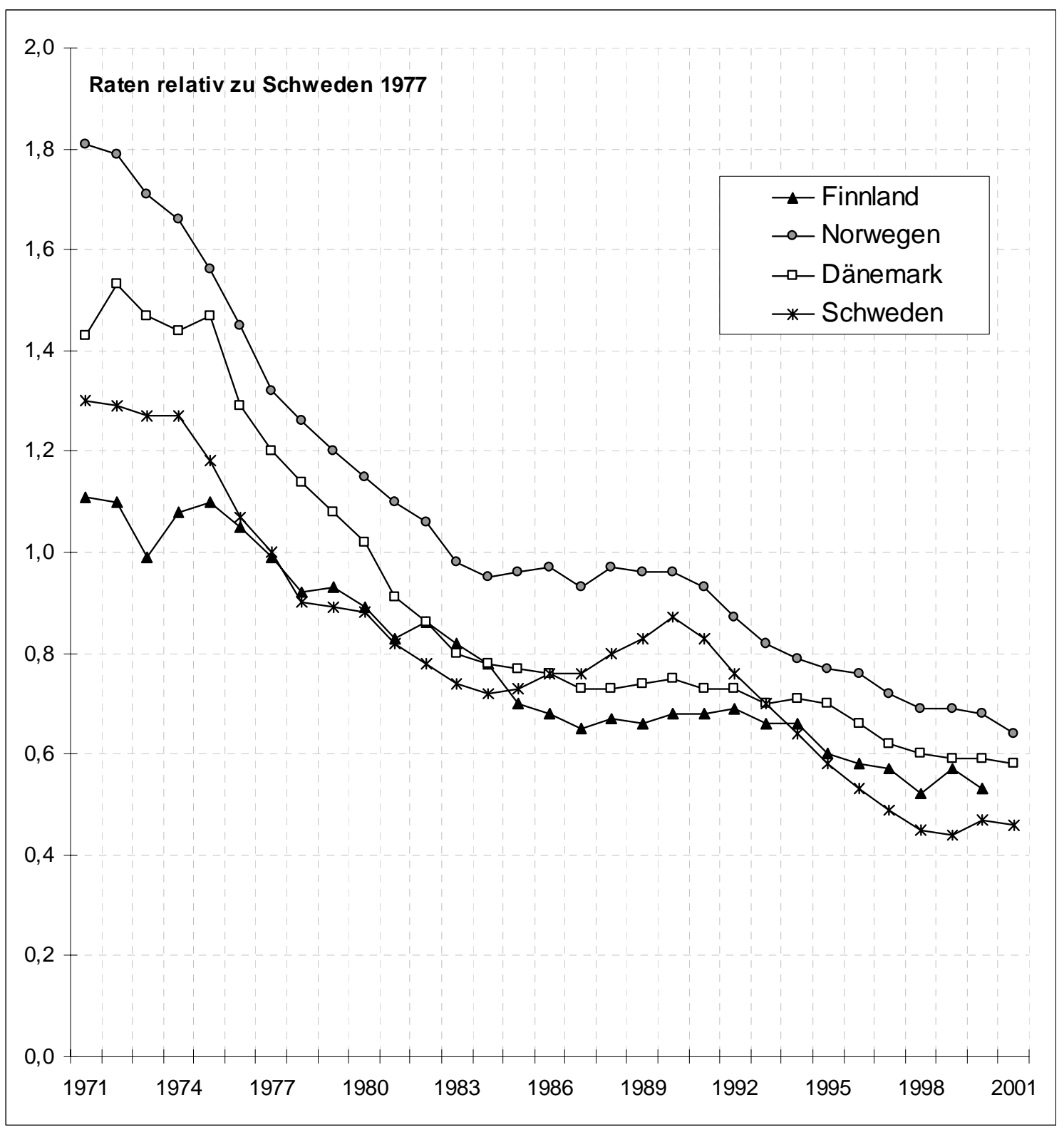


Grafik 2: Standardisierte Erstgeburtenraten kinderloser über 30-jähriger Frauen in den nordischen Ländern

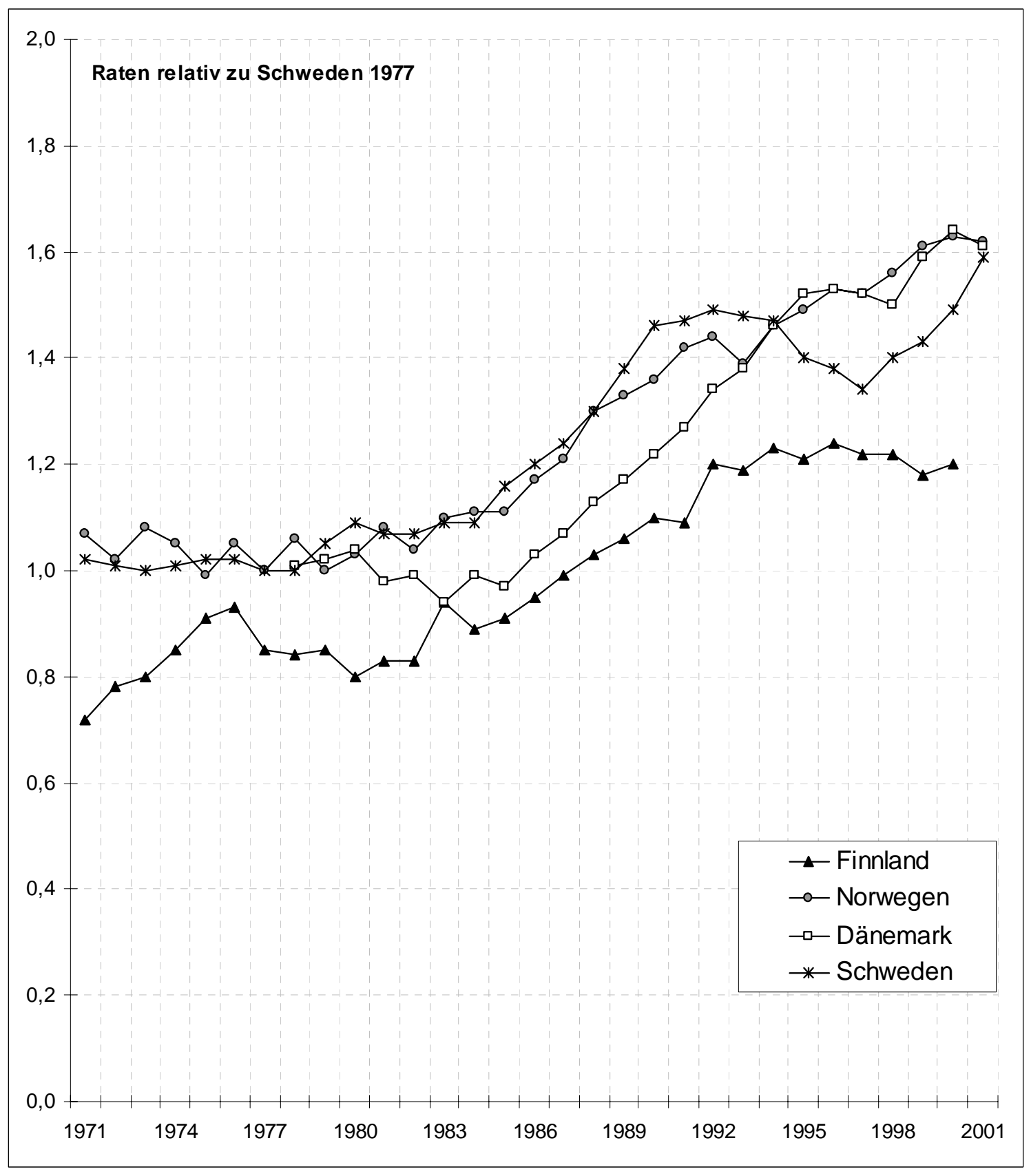


Grafik 3: Standardisierte Zweitgeburtenraten von Müttern mit einem Kind in den nordischen Ländern

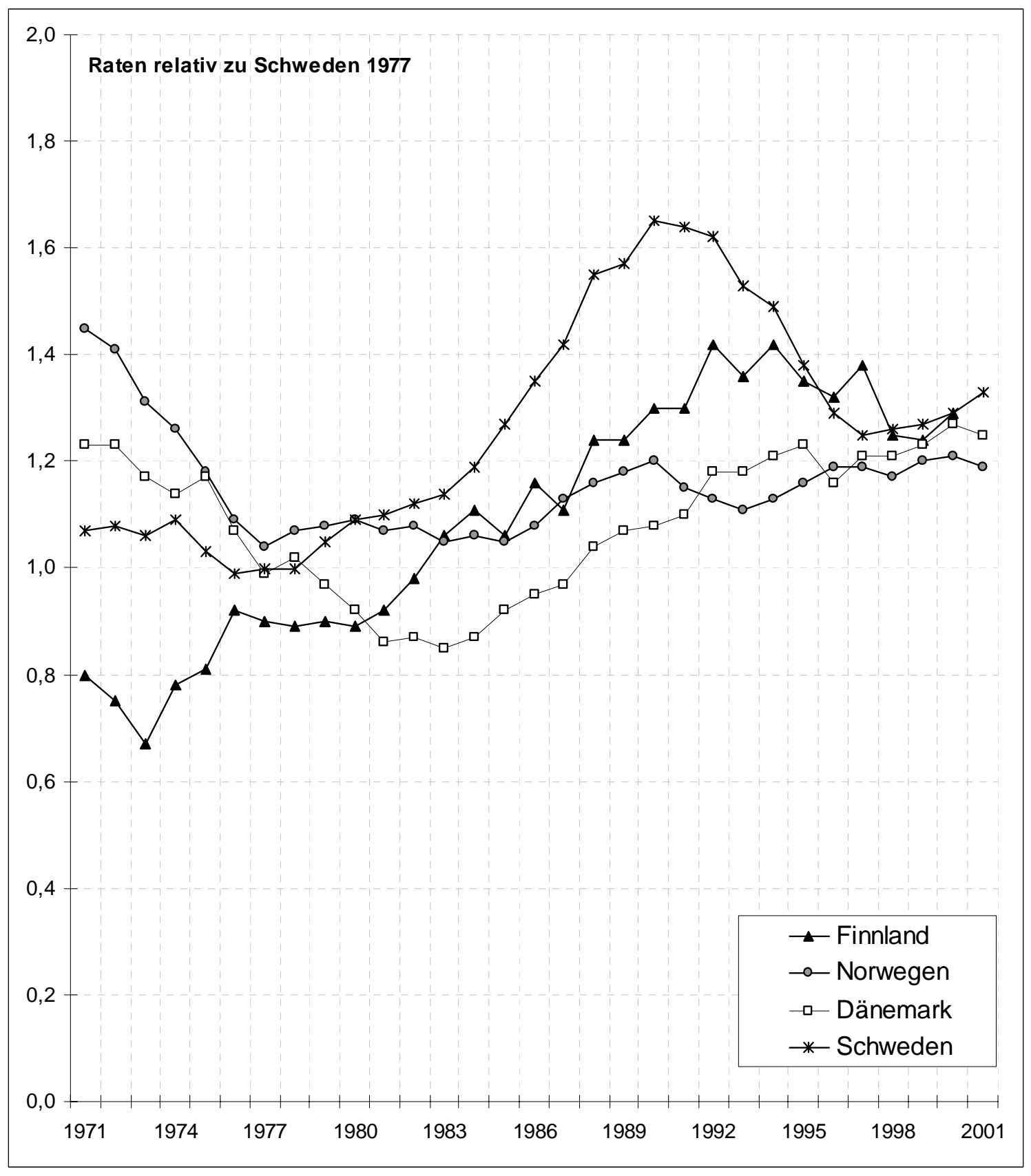

*unter Berücksichtigung des Alters der Mutter und des Alters des ersten Kindes 
Grafik 4: Standardisierte Drittgeburtenraten von Müttern mit zwei Kindern in den nordischen Ländern

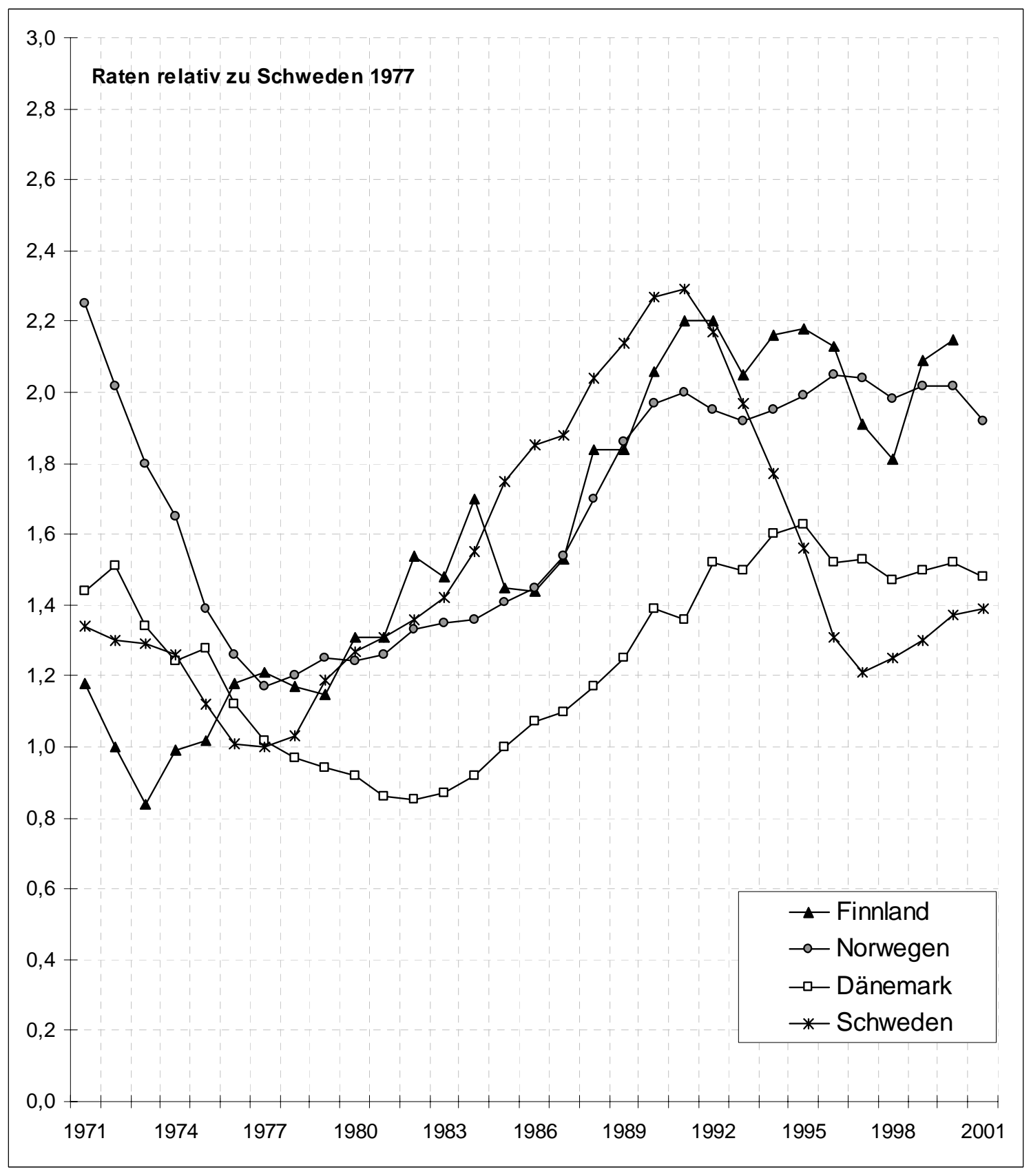

*unter Berücksichtigung des Alters der Mutter und des Alters des zweiten Kindes 
Grafik 5: Altersspezifische standardisierte Zweitgeburtenraten von Müttern mit einem Kind in den nordischen Ländern

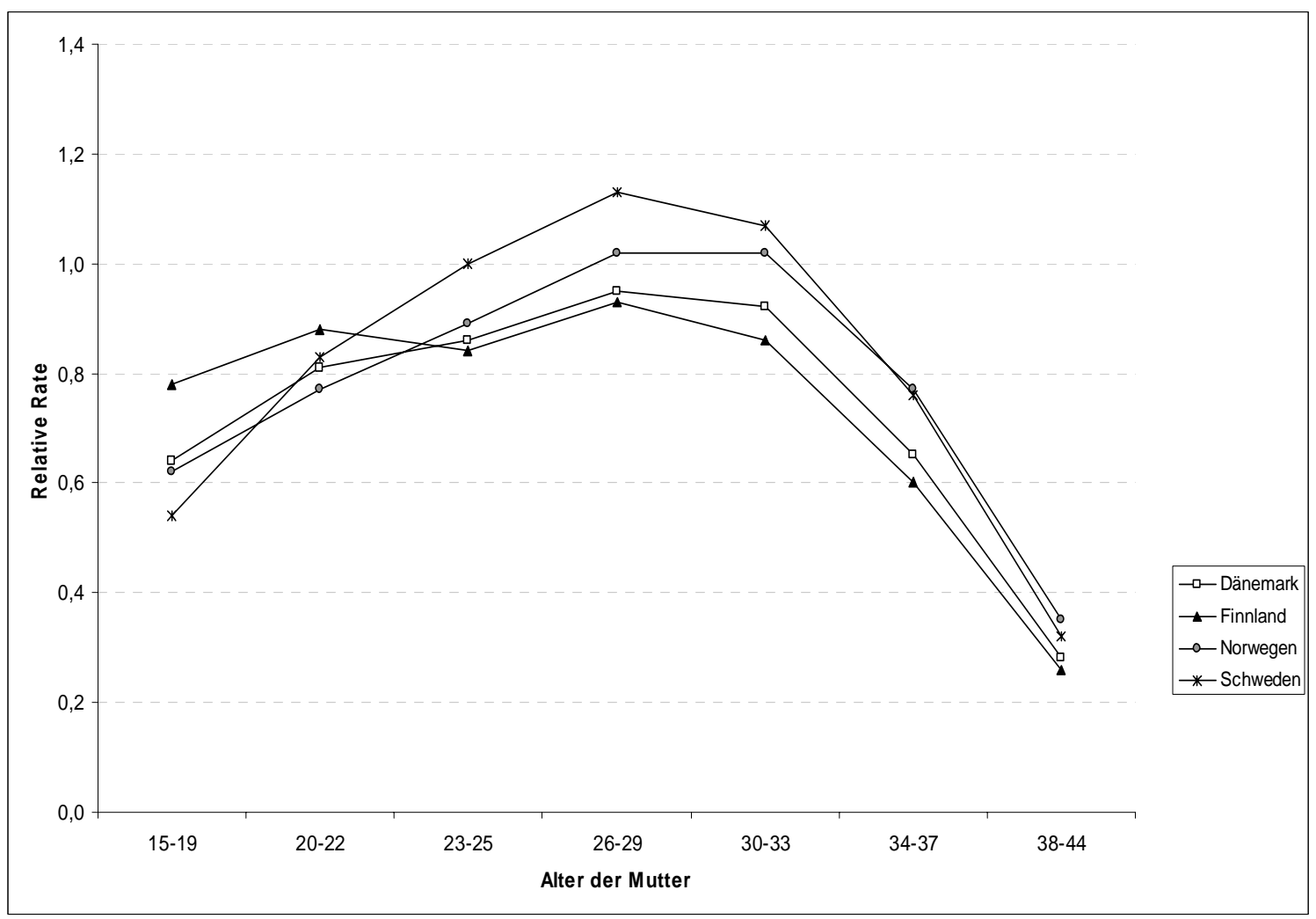

*unter Berücksichtigung des Kalenderjahres und des Alters des ersten Kindes 
Grafik 6: Altersspezifische standardisierte Drittgeburtenraten von Müttern mit zwei Kindern in den nordischen Ländern

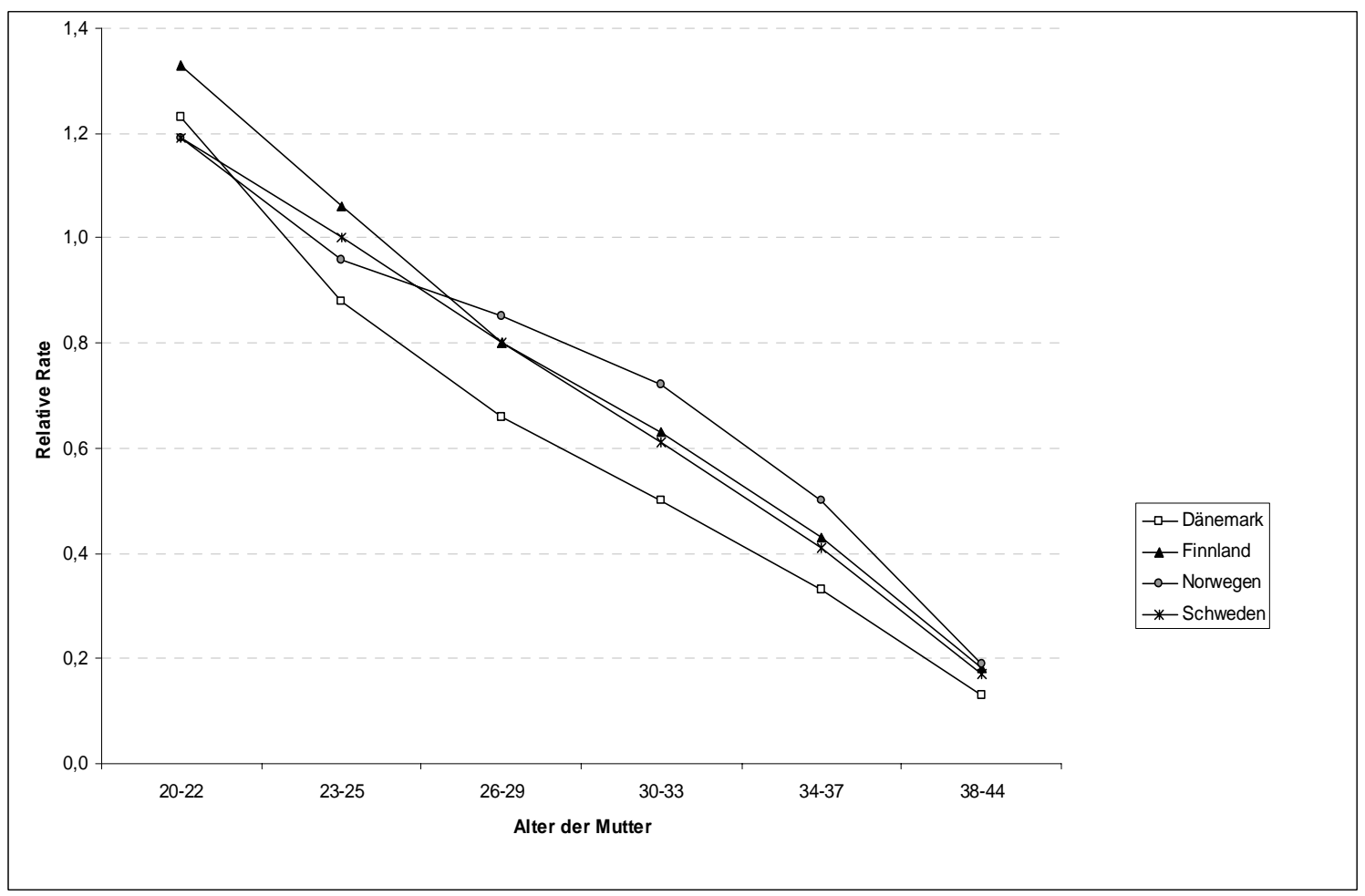

*unter Berücksichtigung des Kalenderjahres und des Alters des zweiten Kindes 
Grafik 7: Kinderlosigkeit nach Ausbildungsrichtung und Ausbildungsgrad: Schwedische Frauen der Geburtenjahrgänge 1955-1959

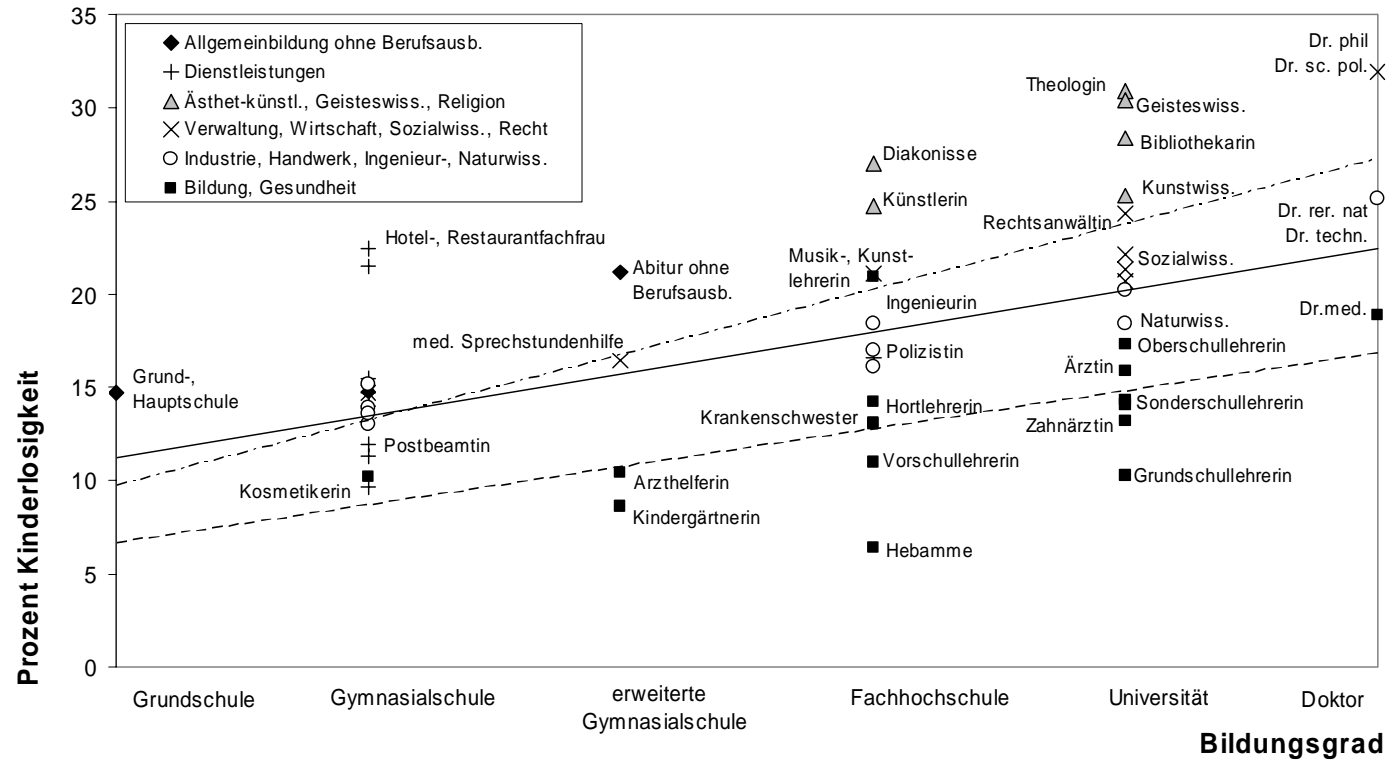

Quelle: Hoem/Neyer/Andersson 2006a 
Grafik 8: Vollständige Fertilität, Ausbildungsrichtung und Ausbildungsgrad: Schwedische Frauen der Geburtenjahrgänge 1955-1959

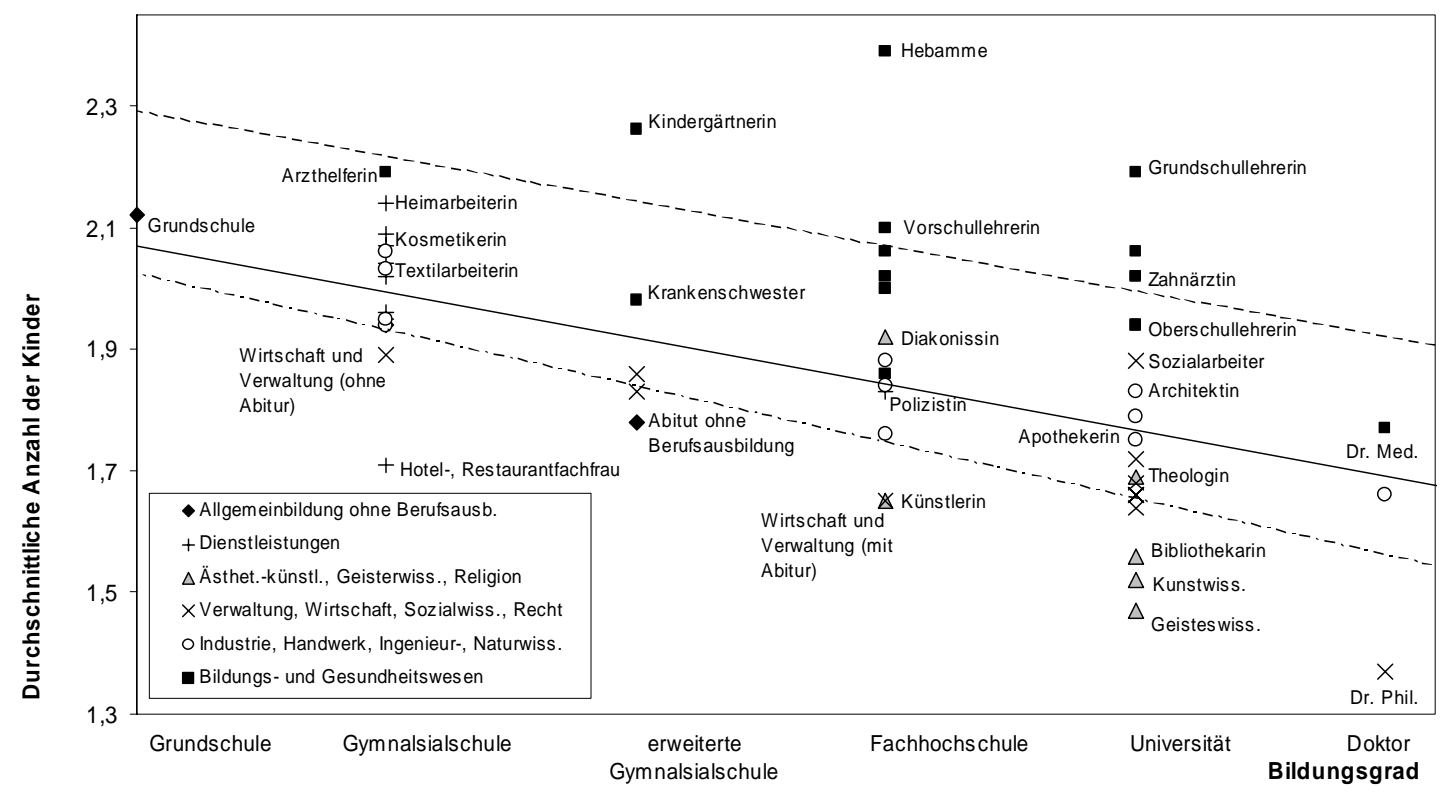

Quelle: Hoem/Neyer/Andersson 2006b 


\section{Grafiken für den Anhang:}

Grafik A1: Gesamtfertilitätsraten: Nordische Länder und Deutschland, 1960 2004

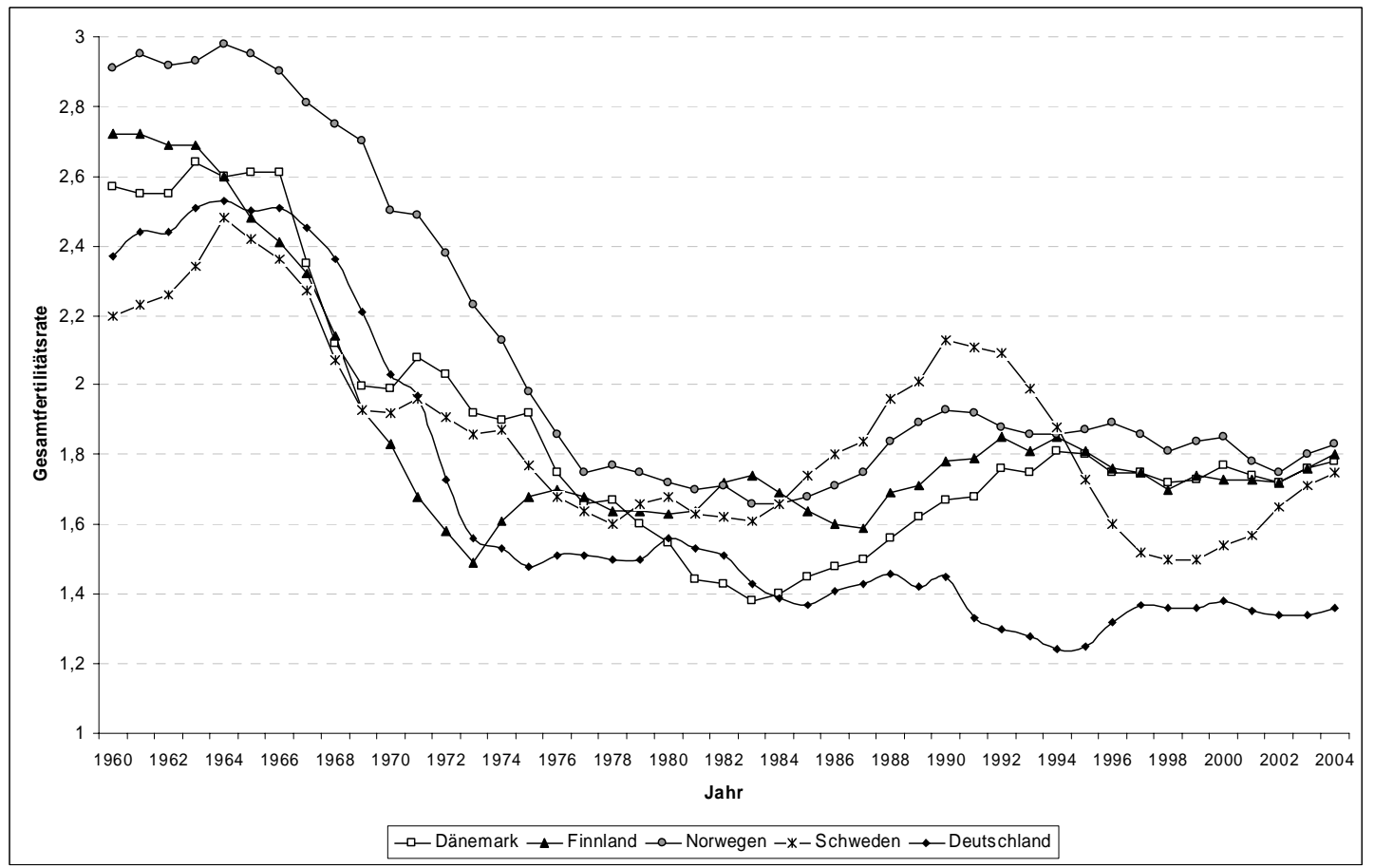

Quelle: Council of Europe 2004. 
Grafik A2: Vollständige Kohortenfertilität von Frauen der Geburtenjahrgänge 1945 und 1960 im Jahre 2002

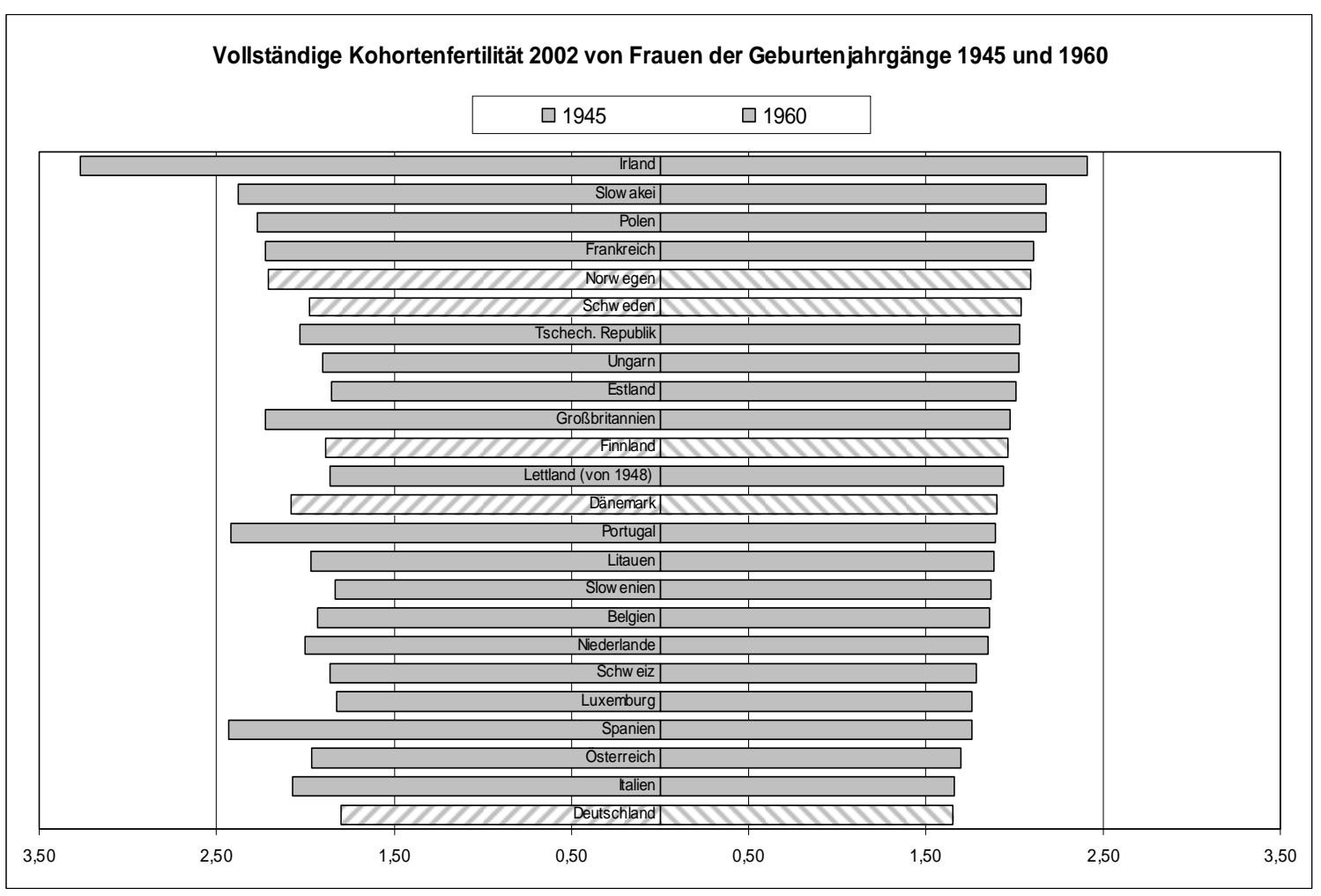

Quelle: Council of Europe (2004) 
Grafik A3: Frauenerwerbsquoten: Nordische Länder und Deutschland 19602004

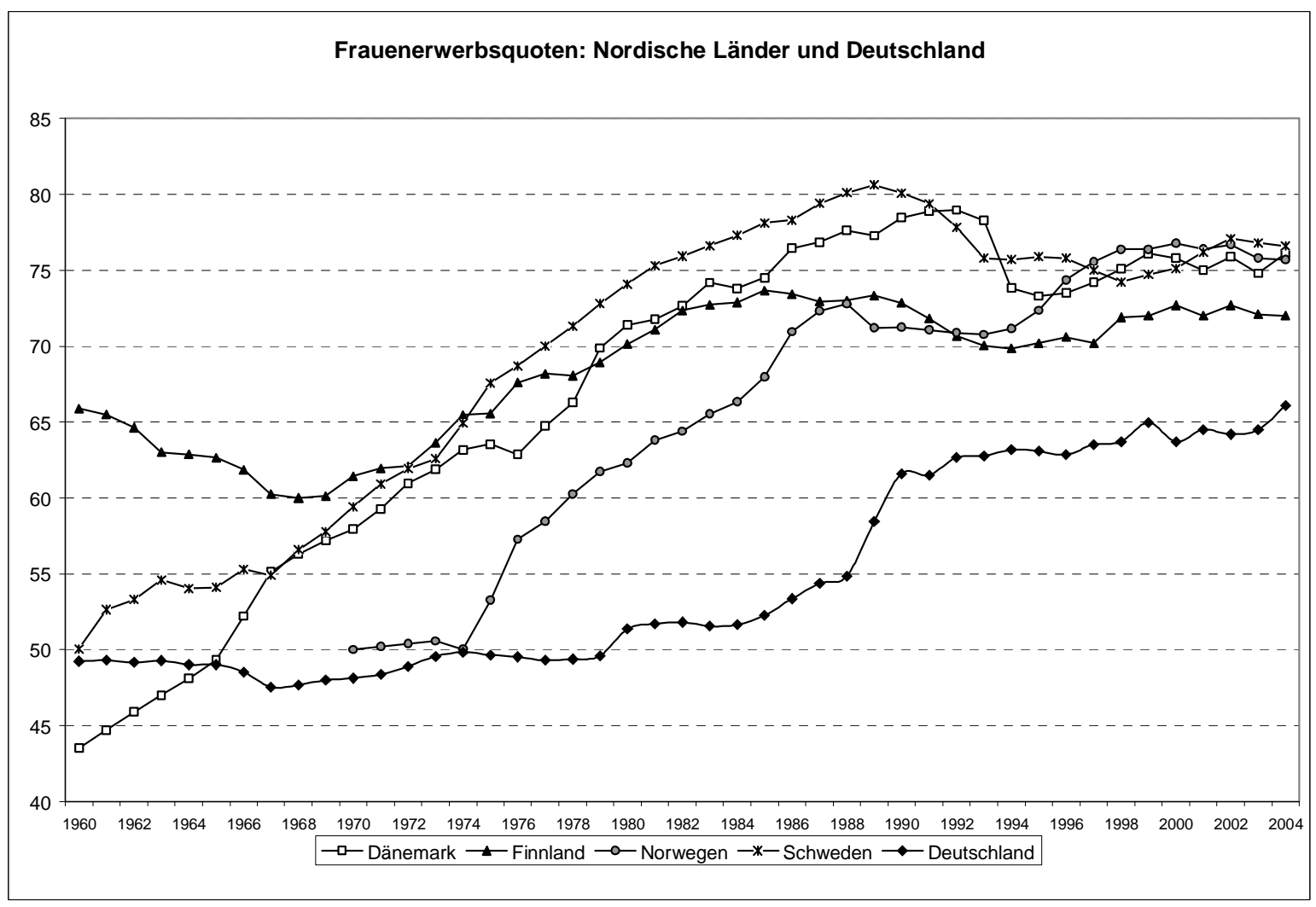

Quelle: OECD 1998; 2004 
Tabelle 1: Elternzeit und Elterngeld in den nordischen Ländern und in Deutschland (2001/2003)

\begin{tabular}{|c|c|c|c|c|c|c|c|c|}
\hline & $\begin{array}{c}\text { Dauer des } \\
\text { Mutterschafts- } \\
\text { urlaubs }\end{array}$ & $\begin{array}{c}\text { Mutterschaftsgeld (in } \\
\% \text { des } \\
\text { vorangegangenen } \\
\text { Einkommens) }\end{array}$ & $\begin{array}{c}\text { Vaterschaftsurlaub } \\
\text { aus Anlass der } \\
\text { Geburt }\end{array}$ & $\begin{array}{l}\text { Dauer der } \\
\text { Elternzeit }\end{array}$ & $\begin{array}{l}\text { Finanzielle } \\
\text { Leistung }\end{array}$ & $\begin{array}{l}\text { Max. Alter } \\
\text { des Kindes }\end{array}$ & Teilzeit & Nur für Vater \\
\hline Dänemark & 18 Wochen & $\begin{array}{c}\max . € 447 \text { pro } \\
\text { Woche oder } \\
100 \% \%^{2}\end{array}$ & 14 Tage & 32 Wochen $^{4}$ & $\begin{array}{c}\text { wie Mutterschaftsgeld; } \\
\text { aliquote Verringerung } \\
\text { bei } 40 \text { bzw. } 46 \text { Wochen } \\
\text { Bezug }\end{array}$ & 9 & ja & nein \\
\hline Finnland & 105 Tage & $43-82^{3}$ & 18 Tage & $\begin{array}{c}158 \text { Tage + } \\
\text { Kinderbetreuungsgeld }\end{array}$ & $\begin{array}{c}43-82 \% \\
\text { einheitlicher Regelsatz }\end{array}$ & $\begin{array}{l}7 \\
3\end{array}$ & ja & 12 Tage $^{5}$ \\
\hline Norwegen & 9 Wochen & 100 & 2 Wochen & $\begin{array}{l}42 \text { bis } 52 \text { Wochen + } \\
\text { Kinderbetreuungsgeld }\end{array}$ & $\begin{array}{c}\text { 100\% für } 42 \text { Wochen } \\
\text { oder } \\
80 \% \text { für } 52 \text { Wochen } \\
\text { einheitlicher Regelsatz }\end{array}$ & 2 & ja & 4 Wochen \\
\hline Schweden & 60 Tage $^{1}$ & 80 & 10 Tage & $\begin{array}{l}390 \text { Tage } \\
+90 \text { Tage }\end{array}$ & $\begin{array}{c}80 \% \\
\text { einheitlicher Regelsatz }\end{array}$ & 8 & ja & 2 Monate \\
\hline Deutschland & 14 Wochen & 100 & nein & 3 Jahre & $\begin{array}{c}\text { Max. } 2 \text { Jahre } \\
\text { einkommensgeprüfter } \\
\text { Regelsatz }\end{array}$ & 8 & ja & nein \\
\hline \multicolumn{9}{|c|}{$\begin{array}{l}\text { Anm:: Zahlen in Kursiv geben jenen Teil der Elternzeit weider, der nur von der Mutter in Anspruch genommen werden kann. } \\
160 \text { Tage der Elternzeit können von der Mutter vor der Geburt als Mutterschaftsurlaub in Anspruch genommen werden. } \\
2 \text { Elterngeld abhängig vom Ausmaß der Erwerbstätigkeit, Lohnhöhe und/oder Mitgliedschaft bei einer Sozialversicherung. Die überwiegende Mehrzahl erhält } \\
\text { das volle Elterngeld von ca. DK } 3.330 \text { (€ } 447 \text { pro Woche) bzw. aufgrund sozialpartnerschaftlicher Vereinbarungen vollen Lohnausgleich. Letzterer wird für } \\
\text { insgesamt } 26 \text { Wochen gewährt (inkludiert Zeit des Mutterschutzes, des Vaterschaftsurlaubs und der Elternzeit). } \\
{ }^{3} \text { Nach Einkommen gestaffelt. } \\
{ }^{4} \text { Kann auf } 40 \text { Wochen (für alle) oder auf } 46 \text { Wochen (für Beschäftigte) ausgedehnt werden. } \\
{ }^{5} \text { Gewährt als zusätzliche Elternzeit für den Vater, falls er mindestens } 2 \text { Wochen der (regulären) Elterzeit in Anspruch nimmt. } \\
\text { Quellen: MISSOC 2001; 2002; 2004; Leira 2002; NOSOSCO 2001; 2002; 2005. OECD Employment Outlook 2001. Deven/Moss 2005. }\end{array}$} \\
\hline
\end{tabular}


Tabelle 2: Kinderbetreuung in den nordischen Ländern: Anteil der institutionell betreuten Kinder an Kindern der jeweiligen Altersgruppe (in \%) in den nordischen Ländern, 1990 - 2003

$\begin{array}{lcccc}\text { Alter } & \text { Dänemark } & \text { Finnland } & \text { Norwegen } & \text { Schweden } \\ 1990 & & & & \\ 0-2 & 48 & 31 & 11 & 29 \\ 3-6 & 73 & 58 & 57 & 64 \\ 0-6 \text { (total) } & 61 & 44 & 33 & 48 \\ 7-10 & 34 & 7 & & 38 \\ & & & & \\ 1995 & & & & \\ 0-2 & 48 & 18 & 22 & 37 \\ 3-6 & 83 & 55 & 61 & 74 \\ 0-6 \text { (gesamt) } & 68 & 39 & 44 & 59 \\ 7-10 & 53 & 5 & & 45 \\ & & & & \\ 2000 & & & & \\ <1 & 15 & 2 & 2 & 0 \\ 1-2 & 77 & 35 & 37 & 60 \\ 3-5 & 92 & 67 & 78 & 86 \\ 0-5 \text { (gesamt) } & 75 & 46 & 52 & 66 \\ 6 & 90 & 67 & & 77 \\ 0-6 \text { (gesamt) } & 77 & 49 & & 68 \\ 7-10 & 63 & 3 & & 51 \\ & & & & \\ 2003 & & & & \\ <1 & 9 & 1 & 2 & 0 \\ 1-2 & 78 & 36 & 44 & 65 \\ 3-5 & 94 & 68 & 85 & 94 \\ 0-5 \text { (gesamt) } & 75 & 46 & 58 & 70 \\ 6 & 90 & 68 & & 83 \\ 0-6 \text { (gesamt) } & 77 & 50 & & 59 \\ 7-10 & 66 & 3 & & \end{array}$

Quelle: NOSOSCO 2002; 2003; 2005 